\title{
The nucleotide sugar UDP-glucose mobilizes long-term repopulating primitive hematopoietic cells
}

\author{
Sungho Kook, ${ }^{1}$ Joonseok Cho, ${ }^{1}$ Sean Bong Lee, ${ }^{2}$ and Byeong-Chel Lee ${ }^{1}$ \\ 1 University of Pittsburgh Cancer Institute and Department of Medicine, Division of Hematology and Oncology, University of Pittsburgh School of Medicine, \\ Pittsburgh, Pennsylvania, USA. ${ }^{2}$ Department of Pathology and Laboratory Medicine, Tulane University School of Medicine, New Orleans, Louisiana, USA.
}

\begin{abstract}
Hematopoietic stem progenitor cells (HSPCs) are present in very small numbers in the circulating blood in steady-state conditions. In response to stress or injury, HSPCs are primed to migrate out of their niche to peripheral blood. Mobilized HSPCs are now commonly used as stem cell sources due to faster engraftment and reduced risk of posttransplant infection. In this study, we demonstrated that a nucleotide sugar, UDP-glucose, which is released into extracellular fluids in response to stress, mediates HSPC mobilization. UDP-glucosemobilized cells possessed the capacity to achieve long-term repopulation in lethally irradiated animals and the ability to differentiate into multi-lineage blood cells. Compared with G-CSF-mobilized cells, UDP-glucose-mobilized cells preferentially supported long-term repopulation and exhibited lymphoid-biased differentiation, suggesting that UDP-glucose triggers the mobilization of functionally distinct subsets of HSPCs. Furthermore, co-administration of UDP-glucose and G-CSF led to greater HSPC mobilization than G-CSF alone. Administration of the antioxidant agent NAC significantly reduced UDP-glucose-induced mobilization, coinciding with a reduction in RANKL and osteoclastogenesis. These findings provide direct evidence demonstrating a potential role for UDP-glucose in HSPC mobilization and may provide an attractive strategy to improve the yield of stem cells in poor-mobilizing allogeneic or autologous donors.
\end{abstract}

\section{Introduction}

Bone marrow transplantation (BMT) is a potentially everlasting curative therapy for hematological diseases such as leukemia, lymphoma, and various types of immunologic disorders. Cancer patients whose own hematopoietic systems have been damaged by chemotherapy or radiation also require BMT.

In recent years, BMT has been replaced by transplantation of mobilized peripheral blood (PB) stem cells (PBSCs), which is safer and less painful for the donor. Functionally, PBSCs engraft better than bone marrow-derived hematopoietic stem progenitor cells (HSPCs) and allow faster recovery of the white blood cell count, thereby reducing the risk of infection in patients during the early posttransplant period (1-3). Accordingly, mobilized cells have become a major source of HSPCs for autologous and allogeneic transplantations. However, the benefits of HSPC mobilization are often offset by potential adverse effects. G-CSF, the most widely used HSPC mobilizer, causes side effects such as spleen enlargement, bone pain, headaches, and propensity for thrombosis $(4,5)$. While it has not yet been investigated in human patients, G-CSFmobilized murine PBSCs also exhibit defective long-term repopulating and self-renewal activity $(6,7)$. Furthermore, patients receiving G-CSF-mobilized PBSCs have an increased incidence of chronic graft-versus-host disease (GVHD) following allogeneic transplantation (8). In addition, because older individuals or patients with Fanconi anemia (FA) show poor HSPC mobilization in response to G-CSF $(9,10)$, it is necessary to tailor mobilization regimens to the individual clinical situation. Patients whose bone marrow has been damaged by extensive chemotherapy and radiation therapy also respond poorly to conventional mobilization regimens. In order to

Conflict of interest: The authors have declared that no conflict of interest exists. Citation for this article: J Clin Invest. 2013;123(8):3420-3435. doi:10.1172/JCI64060. overcome poor mobilization, combinations of G-CSF with different mobilizing agents have been attempted. Notably, a combination of G-CSF with AMD-3100 or cyclophosphamide exerts significant synergistic effects on HSPC mobilization $(11,12)$. However, these combinatorial regimens also increase the risk of adverse events. For example, while the combination of cyclophosphamide with G-CSF may be useful in patients receiving salvage chemotherapy, this regimen often causes neutropenia, fever, and other signs of infection (13). Use of AMD-3100, a synthetic inhibitor of the CXCR4 receptor, is not associated with significant side effects. However, AMD-3100 may be associated with tumor growth (14), although the possible effects of long-term use of AMD-3100 requires further investigation.

Nucleotides, once recognized as mere sources of energy, have recently emerged as important regulators of diverse cellular processes including proliferation, differentiation, and stress responses in vertebrate and invertebrate animals. Upon binding to their cognate receptors, termed purinergic receptors (P2X and $\mathrm{P} 2 \mathrm{Y}$ ), nucleotides trigger intracellular signaling events. The role of nucleotides as regulators of hematopoiesis has become more evident in recent years $(15,16)$. Nucleotides have chemotactic activity and stimulate migration-associated intracellular signaling events such as actin reorganization and mobilization of intracellular calcium (17-19). In line with this, recent progresses have highlighted the physiological significance of extracellular nucleotides in HSPCs: uridine-5' -triphosphate (UTP) is able to chemoattract human CD34+ cells, and ex vivo treatment of human $\mathrm{CD} 34^{+}$cells with UTP enhances the engraftment of HSPCs $(19,20)$. These findings provide a rationale for further preclinical and clinical evaluation of extracellular nucleotides for more robust and efficient stem cell transplantation.

Purines and pyrimidines are released at the site of damage resulting from radiation, stress, or hypoxia and serve as protective signals in concert with other factors (21). In particular, the uridine diphos- 

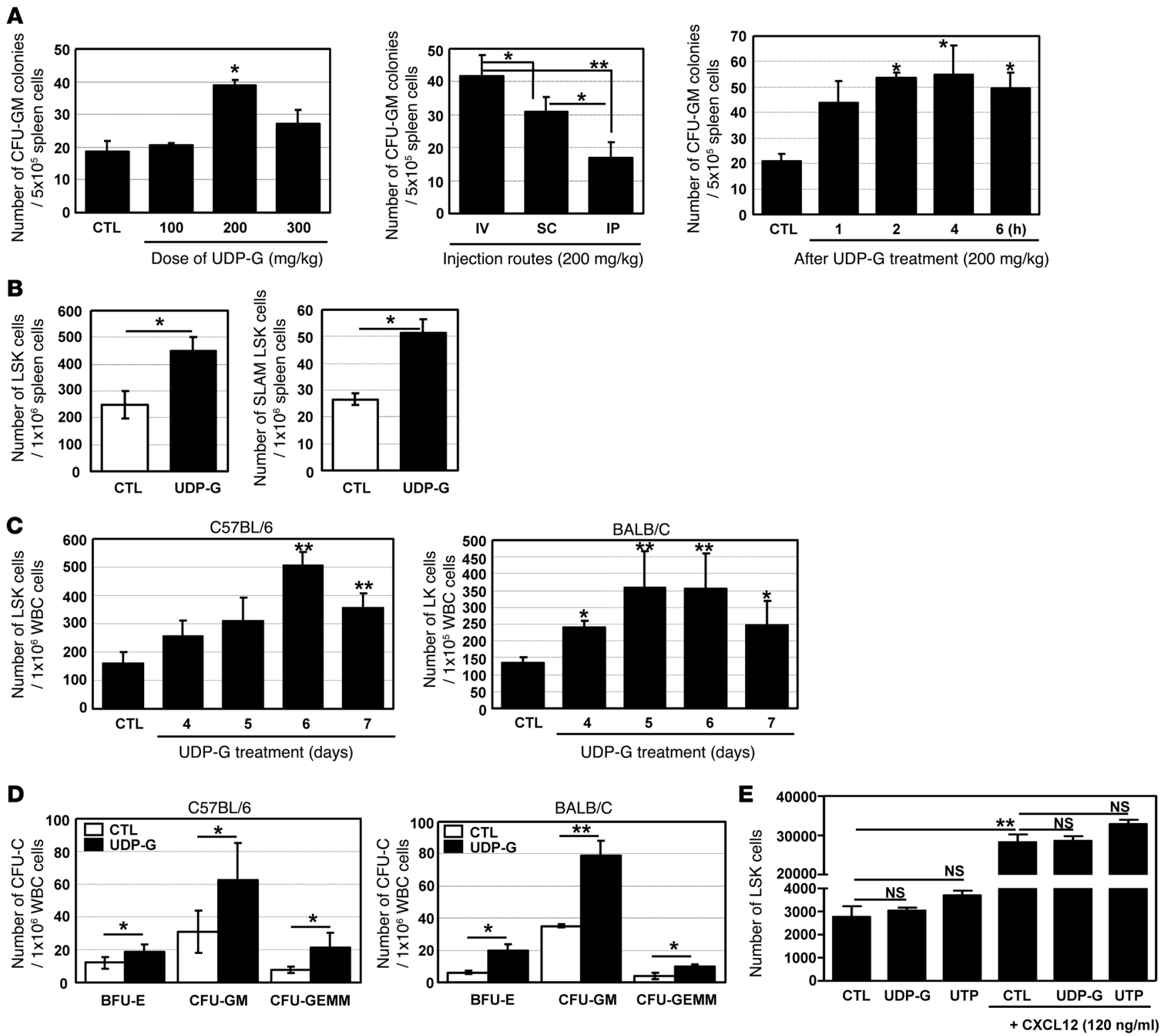

Figure 1

HPC mobilization with UDP-Glc. (A) The effects of doses and injection routes were determined. The effect of UDP-Glc peaked 2-4 hours after UDP-Glc injection, thus PB cells were collected 2-4 hours after the last injection in all experiments unless otherwise stated. (B) B6 mice were injected once daily for 6 days with UDP-Glc (UDP-G, $200 \mathrm{mg} / \mathrm{kg}$ ) or vehicle (CTL). Single-cell suspensions from spleens were stained for LSK and SLAM LSK and the number of HSPCs enumerated. (C) B6 and BALB/C mice were injected daily with UDP-Glc (200 mg/kg), control mice with vehicle. PB was drawn at the indicated time points and LSK cells quantified. Lin ${ }^{-} \mathrm{C}-\mathrm{Kit}^{+}$(LK) subsets were quantified in BALB/C mice, which express low or no Sca-1. (D) B6 and BALB/C mice were treated once daily for 6 days with vehicle or UDP-Glc, PB cells harvested, and CFU-C activity measured. (E) Chemotaxis assays were performed using 5- $\mu \mathrm{m}$-pore filters. Lin- bone marrow cells from B6 mice were placed in the upper well $\left(10^{6} /\right.$ well) and CXCL12 $(120 \mathrm{ng} / \mathrm{ml})$, UDP-Glc $(10 \mu \mathrm{M})$, or UTP $(10 \mu \mathrm{M})$ in lower wells. After 6 hours of incubation, cells were collected from lower wells and LSK cells counted; cell migration was not significantly different at UDP-Glc concentrations of 1 , 10, and $50 \mu \mathrm{M} .{ }^{*} P<0.05,{ }^{* *} P<0.01$. Data are mean \pm SD and reflect 3 independent experiments in $\mathbf{A}, \mathbf{C}$, and $\mathbf{D}$ and 2 independent experiments in $\mathbf{E}$ (duplicate wells/treatment for $\mathbf{D}$ and $\mathbf{E}$ ).

phate-glucose/P2RY14 (UDP-Glc/P2RY14) signaling axis has been shown to be involved in the stress response, such as inflammation and/or mechanical stress $(22,23)$. In addition, UDP-Glc expression was detected at high levels in tumor cells (24), suggesting that it may act as a danger signal for inflammation or tumors.
In the present study, we establish UDP-Glc as a novel physiologic mobilizer of a long-term repopulating subset of HSPCs. UDP-Glc is a naturally occurring substrate for in vivo glycogen synthesis and thus has potential as a new class of small-molecule mobilizers with minimal side effects. 
A

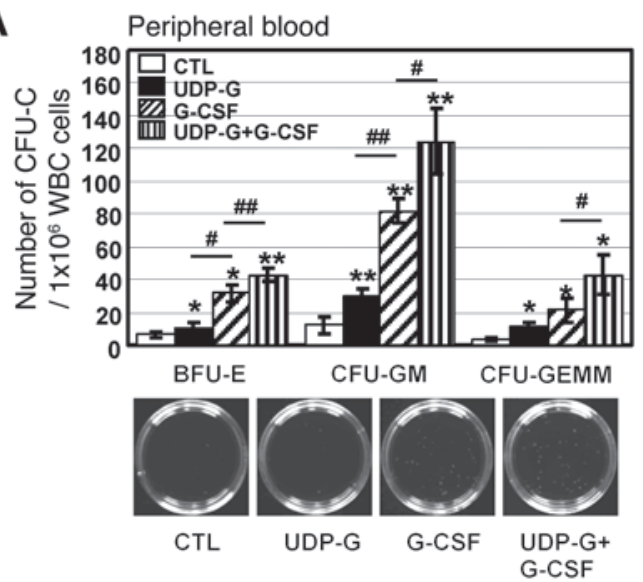

B
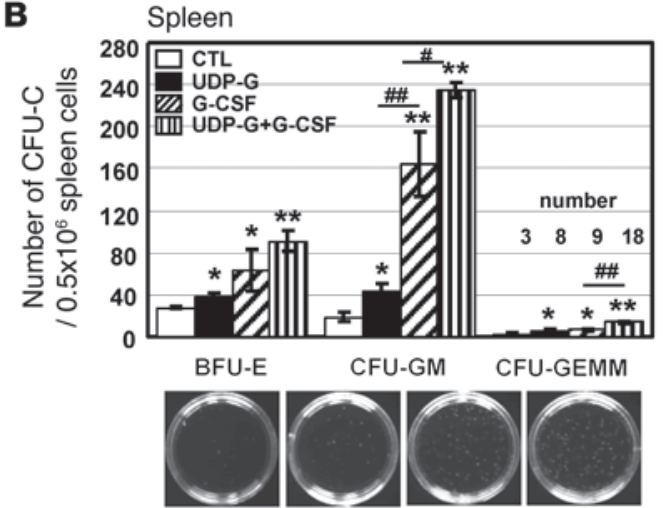

CTL UDP-G G-CSF UDP-G+ G-CSF

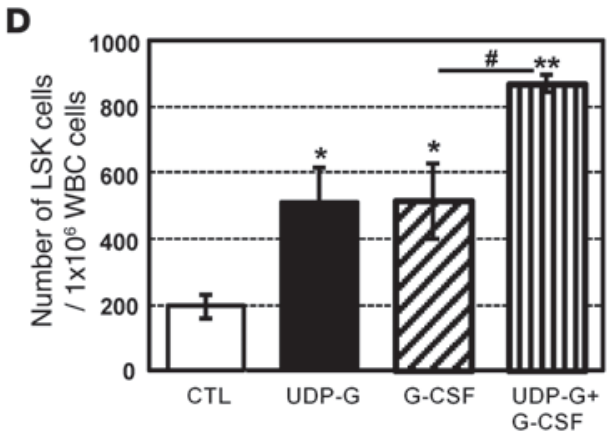

F
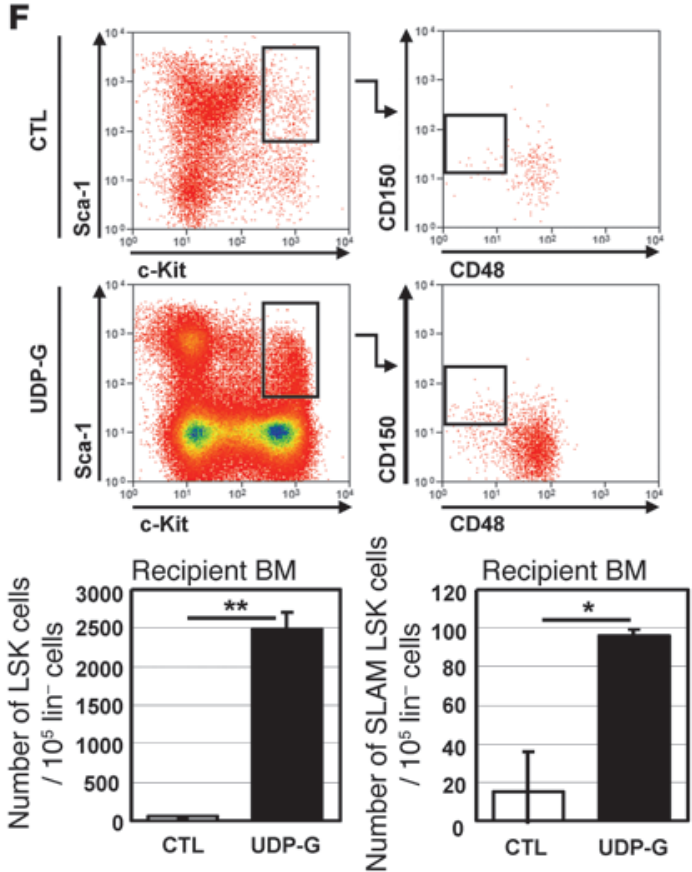

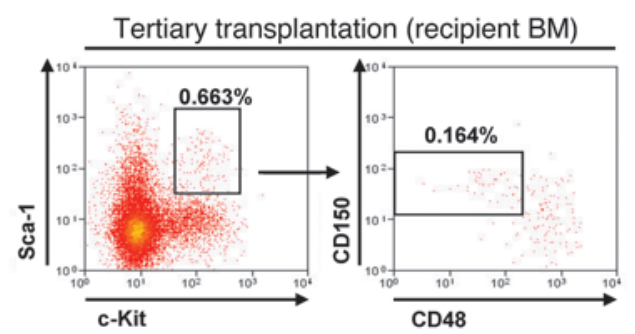




\section{Figure 2}

UDP-Glc mobilizes HSPCs with long-term engraftment potential. (A-D) B6 mice were injected with UDP-Glc (200 mg/kg, 6 days), G-CSF (300 $\mu \mathrm{g} / \mathrm{kg}, 4$ days), G-CSF plus UDP-Glc, or PBS. (A and B) CFU-Cs were counted. (C) Primary stromal cells were prepared as previously described (62). PB cells were harvested and overlaid on irradiated stromal layers in 96-well plates. After 5 weeks, wells containing cobblestone areas were counted as positive wells. (D) Mobilization efficiency was assessed according to the numbers of circulating LSK cells in PB. $n>5$ mice/group. (E) PB cells $\left(1.5-2 \times 10^{6}\right)$ were collected from PBSand UDP-Glc-injected mice and transplanted into lethally irradiated recipient animals (Supplemental Figure 2). The contribution of donor cells was measured by quantifying CD45.1 (vehicle-injected) and CD45.2 (UDP-Glc-injected) cells in recipient PB at the indicated times after transplantation. $(\mathbf{F})$ Sixteen weeks after transplantation, bone marrow cells were analyzed by flow cytometry for LSK and SLAM LSK cells after gating on CD45.1+ (PBS-injected) and CD45.2+ (UDP-Glcinjected) cells. $n=6$ mice/group. (G) Donor-derived SLAM LSK cells $\left(C D 45.2^{+}\right)$were transplantable from primary recipients into irradiated secondary and tertiary recipient mice. Tertiary recipient animals were examined 5-6 weeks posttransplant. PB of recipient animals $(n=5)$ was analyzed for multilineage reconstitution and gated for HSPCs. Data are mean \pm SD and reflect 3 independent experiments in $\mathbf{A}-\mathbf{C}$ (duplicate wells/treatment in $\mathbf{A}$ and $\mathbf{B}$ ). ${ }^{*} P<0.05,{ }^{*} P<0.01$ vs. control; $\# P<0.05, \# \#<0.01$.

\section{Results}

UDP-Glc promotes mobilization of HSPCs. Under stress conditions, HSCs and hematopoietic progenitor cells (HPCs) are mobilized into PB and extramedullary organs such as spleen and liver. Meanwhile, UDP-Glc is known to be released into the extracellular space under stress $(22,23)$. Therefore, we hypothesized that an in vivo administration of exogenous UDP-Glc may mimic stress conditions, which could in turn trigger HSC/HPC mobilization. To investigate this possibility, we injected UDP-Glc into mice and assessed for its ability to mobilize HPCs that are capable of forming colonies (CFU-Cs). Spleen cells from UDP-Glc-treated mice showed an increase in the number of CFU-GM (Figure 1A). In determining optimal dose, UDP-Glc exerted its maximal effects at a dose of $200 \mathrm{mg} / \mathrm{kg}$ body weight (Figure 1A, left panel). Although i.v. administration was superior to s.c. or i.p. (Figure 1A, middle panel), s.c. injection was chosen for further studies to minimize potential complications of i.v. injection and due to its simplicity. The number of spleen-derived CFU-GM was evident within 1 hour after UDP-Glc administration, reaching a peak in approximately 2 to 4 hours (Figure 1A, right panel). Regardless of the assay conditions, we consistently observed an increased number of CFU-GM from the spleen of UDP-Glc-treated mice $(P<0.05)$ (Supplemental Figure 1; supplemental material available online with this article; doi:10.1172/JCI64060DS1). In addition, we observed a statistically significant increase in the number of HSPCs (lineage-Sca- $1^{+} \mathrm{c}-\mathrm{kit}{ }^{+}$ cells, hereafter referred to as LSK cells) in the spleen of UDP-Glctreated mice (Figure 1B, left panel). In particular, UDP-Glc-treated mice exhibited a significant increase in the splenic CD $150^{+} \mathrm{CD} 48^{-}$ LSK cells (hereafter referred to as SLAM LSK cells) (Figure 1B, right panel), which is a very rare and primitive HSC subset (25). The addition of UDP-Glc to the CFU-GM assay did not significantly alter the number of colonies (Supplemental Figure 1).

Since mobilized HSPCs are routinely harvested from the PB in the clinic, we quantified HSPCs in the PB of UDP-Glc-treated mice. There was a notable increase in the frequency of LSK cells in circulation after 6 daily single UDP-Glc injections in B6 mice, which is one of the most difficult mouse strains in which to mobilize cells (ref. 26 and Figure 1C, left panel). The mobilizing effect of UDP-Glc was also evident in BALB/C mice (Figure 1C, right panel), demonstrating a strain-independent effect of UDP-Glc-induced HSPC mobilization. Consistent with this, UDP-Glc treatment led to a statistically significant increase of CFU-Cs (CFU-GM, BFU-E, and CFU-GEMM) in the blood of both B6 and BALB/C mice (Figure 1D). For further study, we used $\mathrm{B} 6$ mice, since not only are these mice congenic for the CD45 isoform, which is useful to track donor cell populations, but also the $\mathrm{KO}$ mice used in the present study to investigate underlying mechanisms had been produced using the B6 background.

It is known that UTP is functionally associated with human HSPC migration and their engraftment $(19,20)$. We thus examined whether UDP-Glc possesses similar activity on mouse HSPCs. SDF-1 $\alpha$ (CXCL12), as anticipated, potently chemoattracted mouse HSPCs (LSK cells) (Figure 1E). However, UTP elicited only a slight increase for mouse LSK cell migration, with no statistical significance in the absence or presence of CXCL12 (Figure 1E).

UDP-Glc mobilizes long-term repopulating HSPCs. G-CSF is the most commonly used cytokine for mobilization of HSPCs in the clinic. We thus determined the mobilizing capability of UDP-Glc in comparison with G-CSF. G-CSF was administered as described in the previous study $(11,12)$. As shown in Figure 2, A and B, the PB and spleen cells from UDP-Glc-treated mice produced significantly less CFU-Cs than counterpart cells from G-CSF-treated mice. We then performed cobblestone area-forming cell assays (CAFCs) to estimate the frequency of more primitive progenitor cells in UDPGlc-mobilized blood. Despite their low in vitro colony-forming capacity, UDP-Glc-mobilized cells displayed high CAFC activity (approximately 10- to 14-fold higher than vehicle-injected group), which was similar to the fold increase observed with G-CSF-mobilized cells (Figure 2C). In addition, UDP-Glc was almost equally potent in mobilizing LSK cells into PB when compared with G-CSF (Figure 2D). Neither phenotypic analysis nor in vitro HPC assays accurately reflect the stem progenitor cell activity in vivo (27). To assess the functional properties of UDP-Glc-mobilized HSPCs in vivo, we performed competitive repopulation assays, in which an equal number of blood cells from control or UDPGlc-treated mice was transplanted into conditioned recipient mice (see schema in Supplemental Figure 2). UDP-Glc-mobilized cells showed a significant repopulation advantage compared with vehicle-treated blood cells over a 4-month period posttransplant (Figure 2E), suggesting the long-term repopulating potential of UDP-Glc-mobilized HSPCs. Of note, no recipients transplanted with control blood cells alone (PB cells from vehicle-injected mice) survived to lethal irradiation. This was primarily due to the fact that HSPCs are present at very low frequencies in steady-state PB, as previously described $(3,28)$. Only when combined with UDPGlc-mobilized cells could recipient animals survive, suggesting that UDP-Glc-mobilized PB contains enough HSPCs that allow the lethally irradiated animal to survive after irradiation.

The maintenance of stem cell pool and generation of functional mature blood cells depend on close interaction with specialized microenvironments or niches in bone marrow (29). Therefore, the engraftment of HSPCs to bone marrow more accurately represents the outcome in clinical protocols. We thus assessed whether donor-derived HSPCs are sustainable in the bone marrow of recipient animals for an extended period after transplantation. Sixteen weeks after transplantation, we could readily detect HSPC populations (LSK and SLAM LSK cells) derived from UDP- 
A
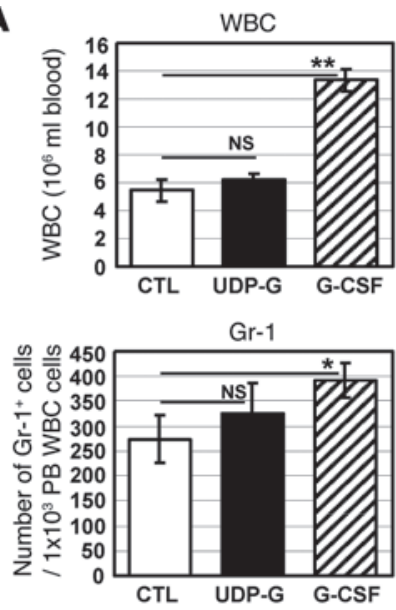
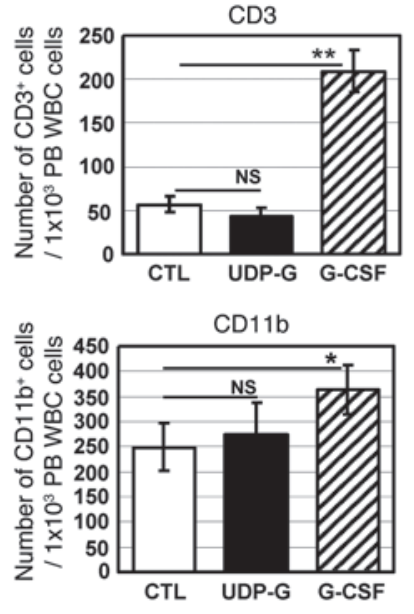

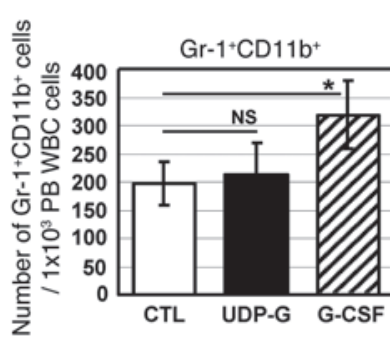

B

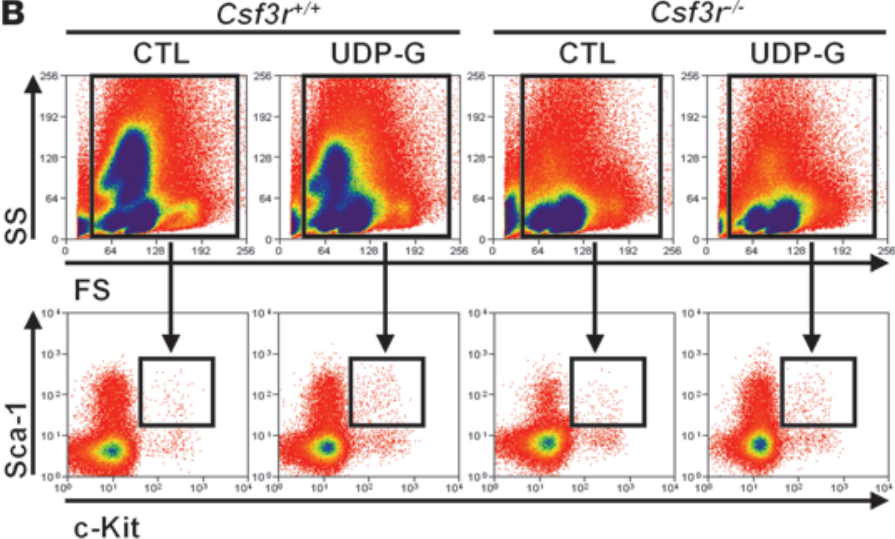

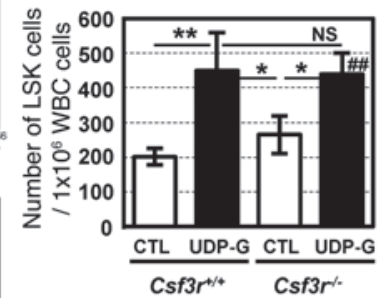

Figure 3

UDP-Glc causes no significant alterations in PB wbc. (A) B6 mice were injected as described in Figure 2A. PB mononuclear cells were collected from each treatment group and stained with indicated antibodies, followed by flow cytometry analysis. Data are depicted as the mean number of wbc per milliliter of blood. For lineage marker-expressing cells, a mean value of the cell number \pm SD obtained from 3 independent experiments is shown. (B) $\mathrm{Csf3r}^{-1-}(\mathrm{KO})$ and WT mice were treated with UDP-GIc or PBS as described above. $n>5$ mice/group. Left: Flow cytometry plots show the gating strategy for identification of LSK cells. PB cells were gated on a forward-scatter/side-scatter (FS/ SS) dot plot. Lin- cells were gated (data not shown), with subsequent gating on $\mathrm{c}-\mathrm{Kit}^{+} \mathrm{Sca}-1^{+}$cells. Right: Mice ( $n>5 /$ group) were individually analyzed for each group. (C) $C s f 3 r^{-1}$ (KO) and WT mice ( $n=5$ /group) were treated as described above. PB was collected and analyzed as described in A. Data are mean \pm SD of 3 independent experiments (A and C). ${ }^{*} P<0.05,{ }^{* *} P<0.01$; $\# P<0.01$ vs. Csfr" ${ }^{++}$CTL.
C
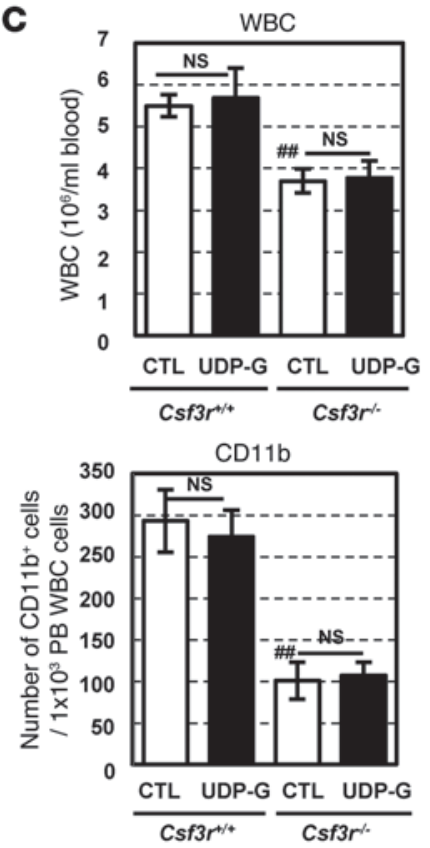
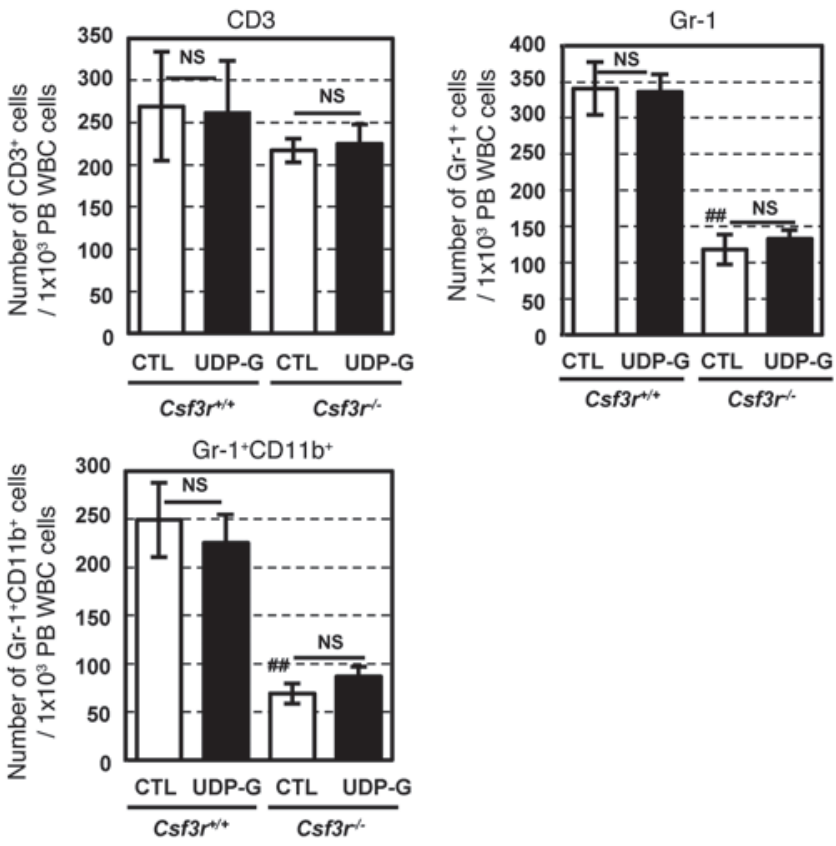

Glc-mobilized cells in the bone marrow of recipient animals (Figure $2 \mathrm{~F}$ ). In contrast, counts of HSPCs derived from vehicle-treated mice were very low or undetectable. To further validate that the phenotypically defined SLAM LSK cells in the primary recip- ient bone marrow were indeed functional HSPCs, SLAM LSK cells were sorted and then subjected to serial transplantation. As shown in Figure 2G, donor-derived SLAM LSK cells were capable of durable multi-lineage engraftment on serial transplantation. 
A

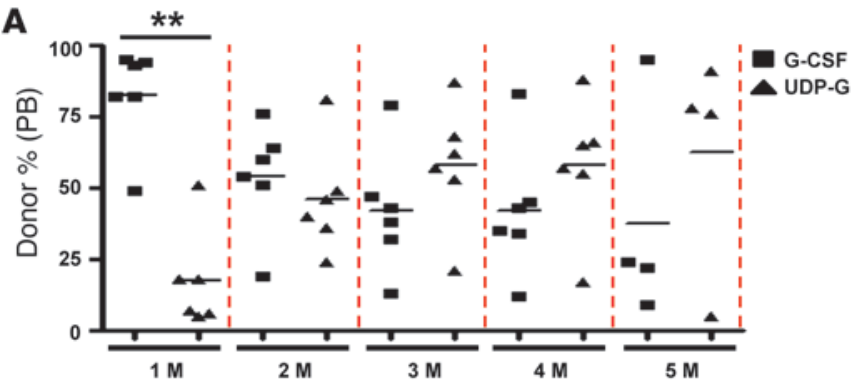

B

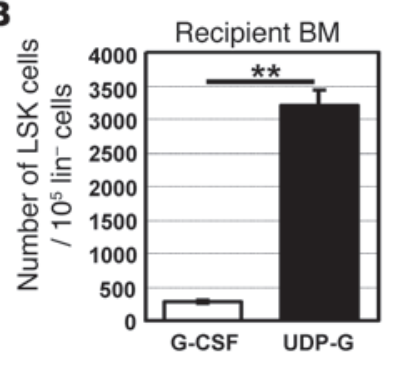

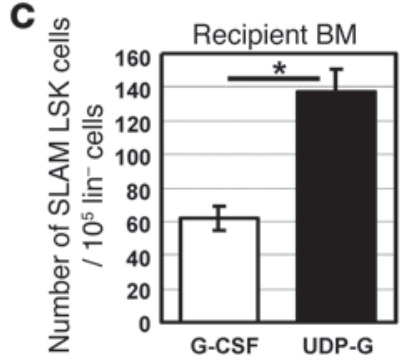

D

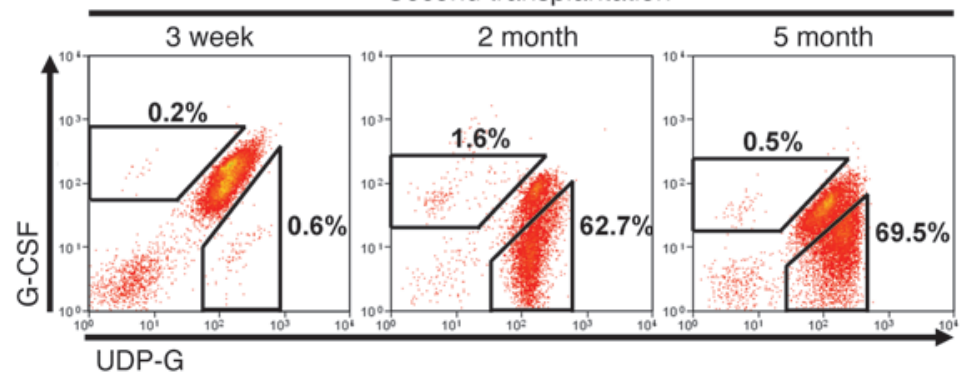

Second transplantation

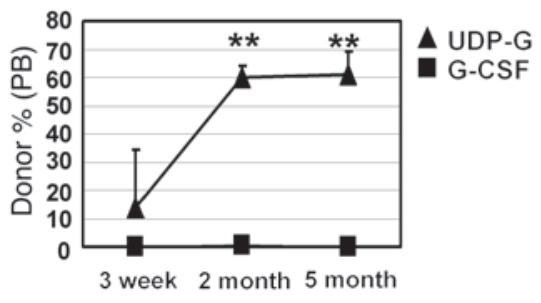

E

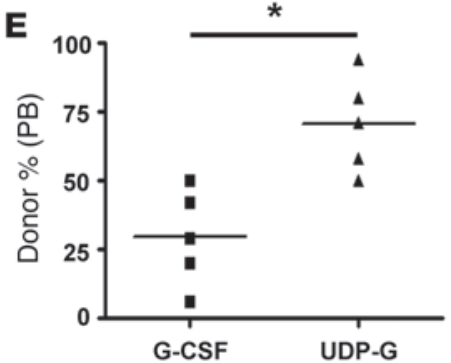

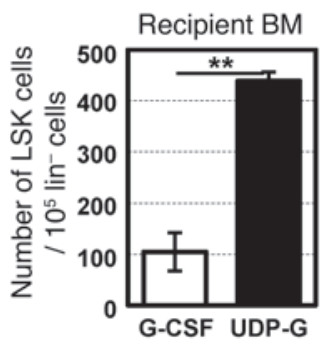

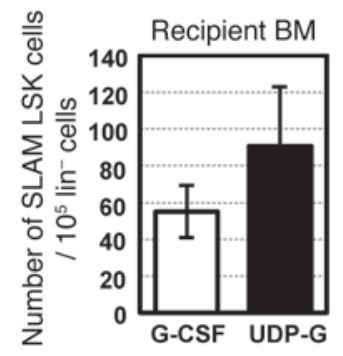

$\mathbf{F}$
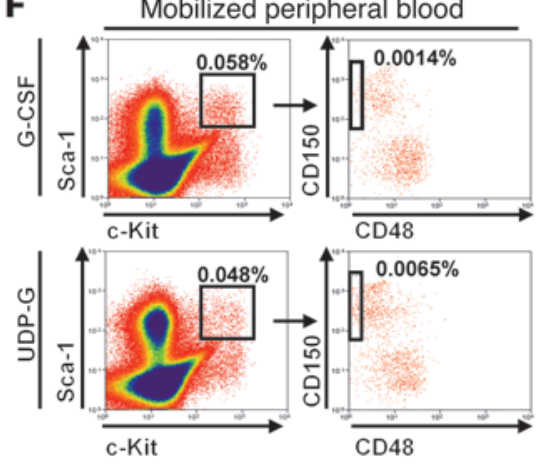

G

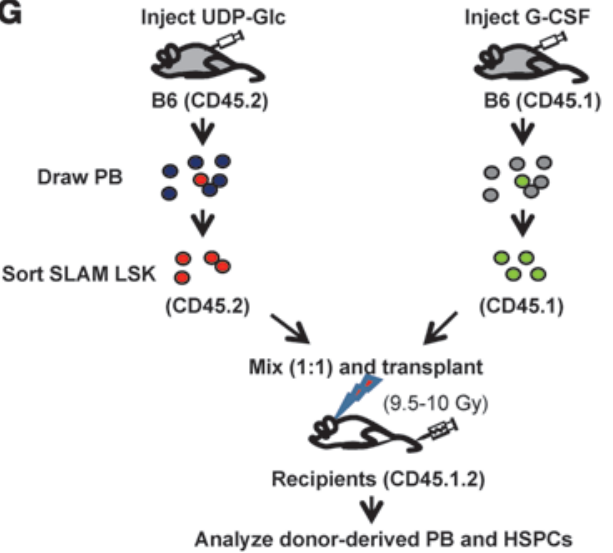

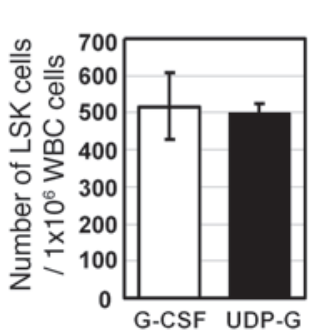
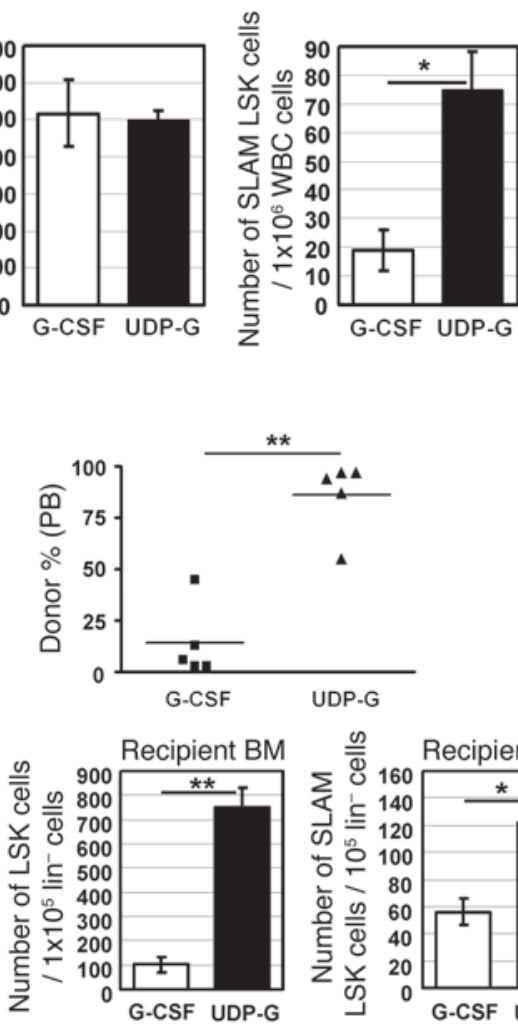

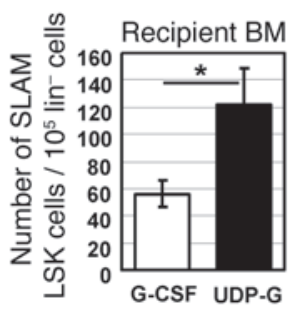

Figure 4

UDP-Glc-mobilized cells display superior long-term repopulating capacity compared with G-CSF-mobilized cells. (A) B6 mice were injected with UDPGlc ( $200 \mathrm{mg} / \mathrm{kg}$, 6 days) or G-CSF (300 $\mu \mathrm{g} / \mathrm{kg}, 4$ days) and competitive repopulation assay performed (Supplemental Figure 2). UDP-Glc- and G-CSF-mobilized cells $\left(2 \times 10^{6}\right.$ each) were transplanted into conditioned animals and contribution of donor cells measured. (B and C) Bone marrow cells were obtained from recipient animals $(n>3)$ and analyzed for LSK and SLAM LSK cells after gating on CD45.1+ (G-CSF) and CD45.2+ (UDP-Glc) cells. (D) Bone marrow cells from primary recipients were sorted based on CD45 expression as described in $\mathbf{A}$ and transplanted into secondary recipients. Donor cell chimerism in recipient mice was analyzed at the indicated times. (E) Bone marrow SLAM LSK cells of primary recipients were used for serial transplantation into secondary and tertiary recipients and engraftment analyzed in tertiary recipients $(n=5)$. (F) B6 mice $(n=4-5)$ were injected with UDP-Glc or G-CSF as in $A$ and PB cells analyzed for LSK and SLAM LSK cells. Data pooled from 2 independent experiments. (G) B6 mice were injected with UDP-Glc $(n>15)$ or G-CSF $(n>12)$ as in A. Mononuclear cells obtained from PB were stained for SLAM LSK cells, which were transplanted into primary recipients, then bone marrow cells transplanted into lethally irradiated secondary and tertiary recipients, and engraftment was analyzed in tertiary recipients (as in $\mathbf{E} ; n=5$ ). Data are mean $\pm \mathrm{SD}$. ${ }^{\star} P<0.05,{ }^{* *} P<0.01$. 
A

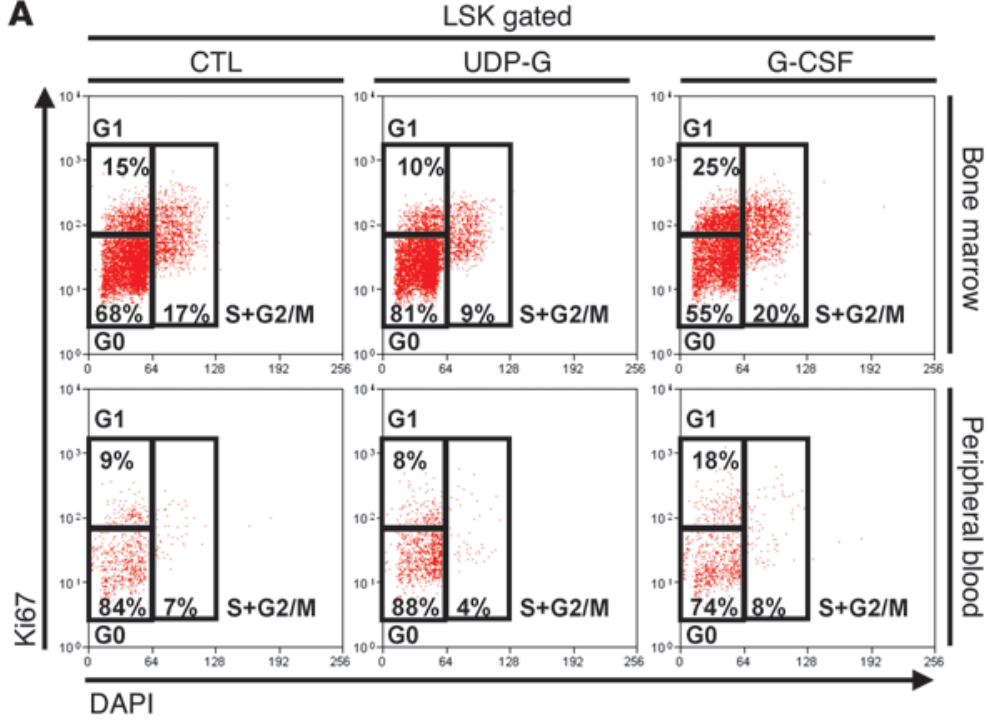

B
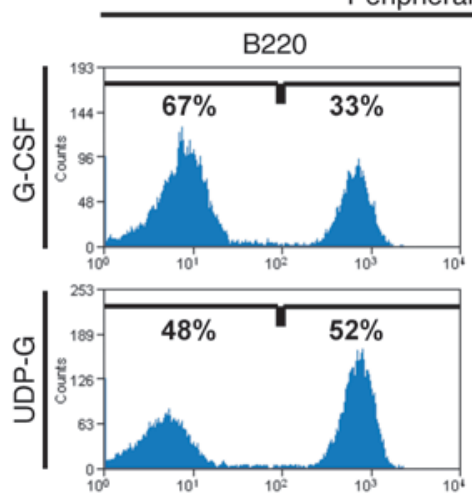

Peripheral blood cells
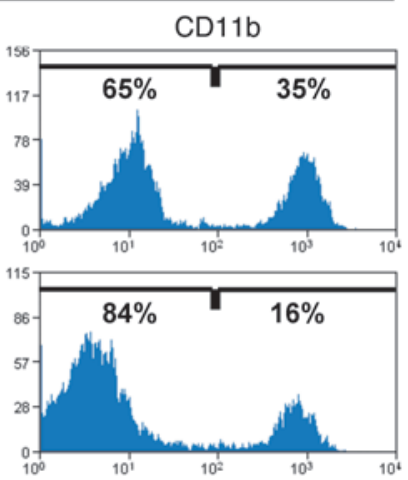
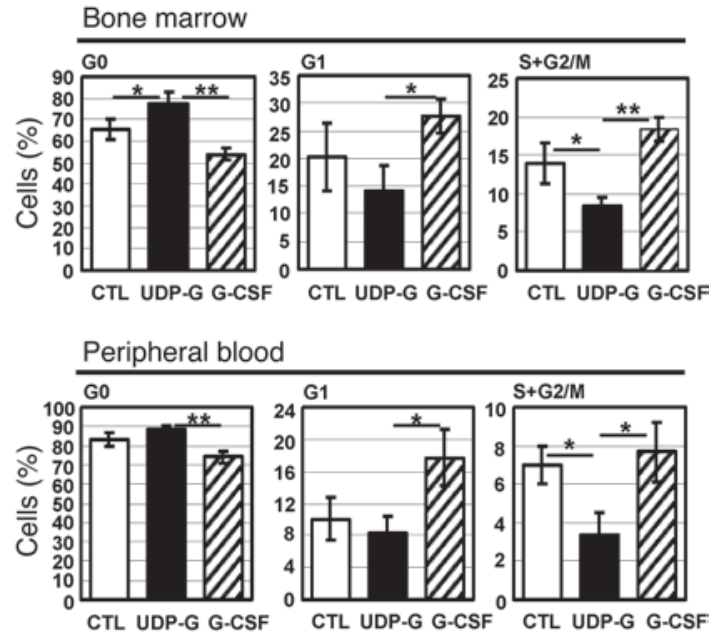

C

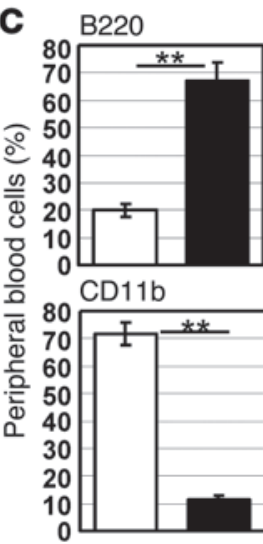

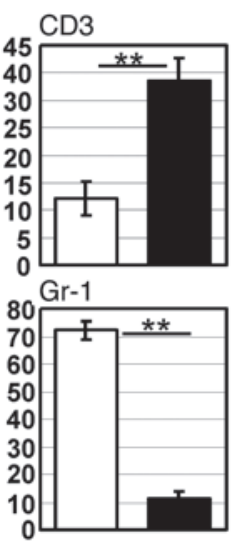

Figure 5

UDP-Glc mobilizes distinct subsets of HSPCs in comparison with G-CSF. (A) The effect of UDP-Glc on cell cycle status of HSPCs was evaluated using LSK cells from vehicle- or UDP-Glc-injected mice. Mice were injected once daily with UDP-Glc or PBS for 6 days as described above. The bone marrow and PB samples were pooled from each group $(n>4)$ and stained for Ki67 and DAPI. LSK cells were pregated and further analyzed for their cell cycle status. Left: The proportions of cells in the respective cell cycle phases are indicated in percentages. Right: Data are mean \pm SD of 3 independent experiments with 4 to 5 mice per group. (B) Recipient animals were transplanted as described in Figure 4A. PB cells from recipients $(n>3)$ were analyzed at 3-4 months posttransplant. The differentiation potential of UDP-Glc- and G-CSF-mobilized cells was determined using CD11b and B220 as markers of myeloid and lymphoid lineages, respectively. (C) Lineage analysis of donor cells in the blood of recipients. At 5 months posttransplantation, PB mononuclear cells of recipient mice were stained with the indicated lineage markers. Data are presented as the percentage of gated cells positive for each lineage marker. Data are mean \pm SD of 2 independent experiments with 3 mice per group. ${ }^{*} P<0.05,{ }^{\star \star} P<0.01$.

These results demonstrate that the SLAM LSK subsets detected in the primary recipient bone marrow were functional HSPCs.

Of note, despite the significant increases in peripheral HSPCs, there was no significant change in the number of wbc in mice treated with UDP-Glc (Figure 3A). Importantly, UDP-Glc-treated mice did not show a significant increase in the number of $\mathrm{CD}^{+}, \mathrm{Gr}-1^{+}$, and $\mathrm{CD}_{11} \mathrm{~b}^{+}$cells in their $\mathrm{PB}$, which is contrary to those observed in mice treated with G-CSF (Figure 3A). A similar pattern in response to UDP-Glc was observed in G-CSFR-deficient mice ( $\left.\mathrm{Cs} f 3 \mathrm{r}^{-} / \mathrm{-}\right)$. Despite the fact that G-CSFR-deficient mice are neutropenic at baseline (30), UDP-Glc was still able to induce a statistically significant mobilization of LSK cells in Csf3r-- mice (Figure 3B). Meanwhile, the numbers of peripheral $\mathrm{CD}_{11} \mathrm{~b}^{+}, \mathrm{Gr}-1^{+}$, and $\mathrm{CD}^{+}$cells were minimally affected in UDP-Glc-treated Csf3r/- mice (Figure 3C).
UDP-Glc-mobilized cells demonstrate superior long-term repopulation activity compared with G-CSF-mobilized cells. Next we compared the HSPC mobilizing capability of UDP-Glc with that of G-CSF using a competitive repopulation assay. At 1 month following transplantation, G-CSF-mobilized cells displayed a considerable competitive advantage over UDP-Glc-mobilized cells (Figure 4A). However, UDP-Glc-mobilized cells began to gain the ability to compete with G-CSF-mobilized cells at 2 months posttransplant. Notably, UDP-Glc-mobilized cells became dominant and out-competed G-CSF-mobilized cells starting at 3 months posttransplant (Figure $4 \mathrm{~A}$ ) and sustained their competitive advantage thereafter.

We assessed whether this was due to preferential engraftment of the recipients' bone marrow niches by UDP-Glc-mobilized cells. To this end, we analyzed the bone marrow of primary recip- 
A
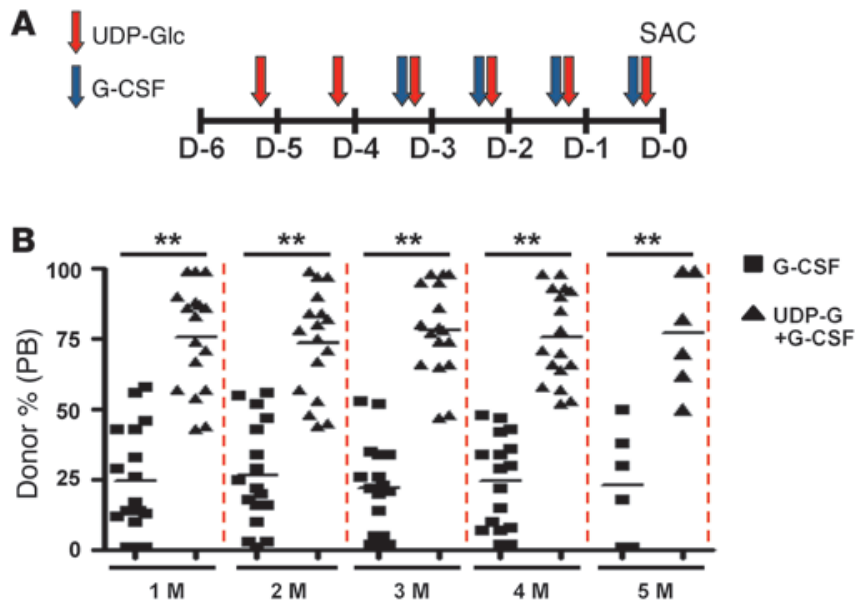

C

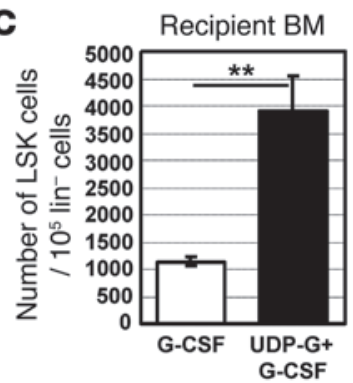

D

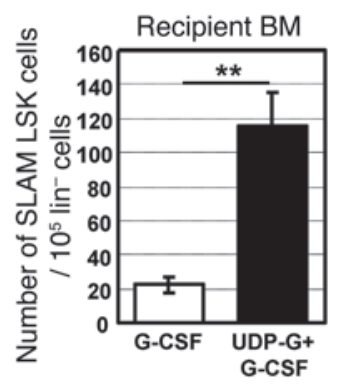

ient animals at 18 weeks after transplantation and found that significantly higher proportions of LSK and SLAM LSK cells in recipient bone marrow were derived from UDP-Glc-treated mice (Figure 4, B and C), indicating that UDP-Glc-mobilized cells achieved higher levels of long-term engraftment than did G-CSF-mobilized cells.

In order to further compare the long-term repopulation abilities of UDP-Glc- and G-CSF-mobilized HSPCs, we performed serial transplantation experiments under competitive settings. Primary recipients (CD45.1.2) were transplanted with UDP-Glcmobilized (CD45.2) and G-CSF-mobilized (CD45.1) PB cells, as shown in Figure 4A. At 2 to 3 months following transplant, bone marrow cells from primary recipients were sorted based on their expression of CD45. A mixture of equal numbers of CD45.1 (derived from G-CSF mobilization) and CD45.2 (derived from UDP-Glc mobilization) bone marrow cells were then transplanted into lethally irradiated secondary recipients (CD45.1.2). G-CSF mobilization-derived cells were out-competed by the cells derived from UDP-Glc mobilization in the secondary recipients over the whole posttransplantation period (Figure 4D). To confirm that the phenotypically defined bone marrow SLAM LSK cells in primary recipients (Figure 4C) is correlated with stem cell function, we sorted SLAM LSK cells from the bone marrow of primary recipient animals and then tested their ability to support serial transplantation. When equal numbers of sorted bone marrow SLAM LSK cells were subjected to competitive serial transplantation, CD $45.2^{+}$SLAM LSK cells (derived from the bone marrow of recipients transplanted with UDP-Glc-mobilized $\mathrm{PB}$ ) exhibited superior engraftment potential as compared with CD45.1 ${ }^{+}$SLAM LSK cells (derived from the bone marrow of recipients transplanted with G-CSF-mobilized PB) (Figure 4E).

\section{Figure 6}

A combination of UDP-Glc and G-CSF has an improved mobilization efficacy over the use of each agent alone. (A) Combinatorial administration schedule: G-CSF was injected daily for 4 consecutive days. UDP-Glc was injected daily for 6 consecutive days. Mice were sacrificed (day 0; SAC) and blood cells analyzed for HSPC activity. (B) Mice were treated as described in A. PB cells were collected from G-CSF- and UDP-GIc/G-CSF-injected mice and transplanted in equal numbers into lethally irradiated recipient animals. The contribution of donor cells in the PB of recipient animals was assessed at the indicated times. ( $\mathbf{C}$ and $\mathbf{D}$ ) Eighteen weeks after transplantation, bone marrow cells were obtained from recipient animals $(n=5)$ and analyzed by flow cytometry for LSK (C) and SLAM LSK (D) cells. Data are mean \pm SD. ${ }^{* *} P<0.01$.

The preferential engraftment of long-term repopulating cells with UDP-Glc-mobilized cells may indicate the possibility that UDP-Glc mobilizes a more primitive subset of HSPCs such as SLAM LSK cells than G-CSF. UDP-Glc promoted LSK cell mobilization into the PB, with efficacy similar to that of G-CSF $(0.048 \%$ vs. $0.058 \%$ ) (Figure 4F). However, UDP-Glc-mobilized LSK cells contained a significantly higher proportion of SLAM LSK cells compared with those of G-CSF-mobilized LSK cells $(0.0065 \%$ vs. $0.0014 \%$ ) (Figure 4F). To directly compare HSC activity of UDPGlc- and G-CSF-mobilized PBSCs, we purified SLAM LSK cells from the PB of mice treated with G-CSF or UDP-Glc and evaluated their HSC activity in a serial transplantation assay. UDPGlc-mobilized PB SLAM LSK cells had greater HSPC activity than G-CSF-mobilized counterpart cells, as evidenced by their superior ability to reconstitute the hematopoietic system of irradiated recipients in serial transplantation assays (Figure 4G). Taken together, these results strongly suggest that UDP-Glc-mobilized HSPCs have enhanced self-renewal capacity when compared with G-CSF-mobilized HSPCs.

UDP-Glc mobilizes distinct subsets of HSPCs in comparison with G-CSF. It is known that G-CSF administration promotes cell cycle entry of quiescent bone marrow HSC in both mouse and baboon (31). Unlike G-CSF, however, UDP-Glc did not appear to function as a potent mitogen for bone marrow HSPCs (Supplemental Figure 1). Therefore, we hypothesized that UDP-Glc releases HSPCs from the niche without causing a significant disruption in cell cycle quiescence, and this may improve the long-term engraftment ability of UDP-Glc-mobilized HSPCs. Indeed, compared with bone marrow LSK cells isolated from G-CSF-treated mice, the bone marrow LSK cells isolated from UDP-Glc-treated mice contained a significantly higher proportion of cells in G0 and lower proportion in the G1, S, and $\mathrm{G} 2 / \mathrm{M}$ phases (Figure $5 \mathrm{~A}$, top right panel). We then analyzed the cell cycle profiles for UDP-Glc-mobilized PB LSK cells. In comparison to G-CSF-mobilized PB LSK cells, UDP-Glc-mobilized PB LSK cells showed an increased distribution in quiescent (G0) fraction and concomitant decreases in $\mathrm{G} 1, \mathrm{~S}$, and $\mathrm{G} 2 / \mathrm{M}$ fractions (Figure 5A, bottom right panel).

It is also noteworthy that UDP-Glc-mobilized cells, as compared to G-CSF-mobilized counterparts, exhibited a differentiation pattern skewed toward the lymphoid lineage in recipient mice (Figure 5B). Of note, with more time elapsed from transplantation, the lineage skewing became more prominent: UDP-Glc-mobilized HSPCs became more skewed toward lymphoid cells, with a longer posttransplantation period (Figure 5C). These data support the notion that UDP-Glc mobilizes a functionally distinct subset of HSPCs. 
A

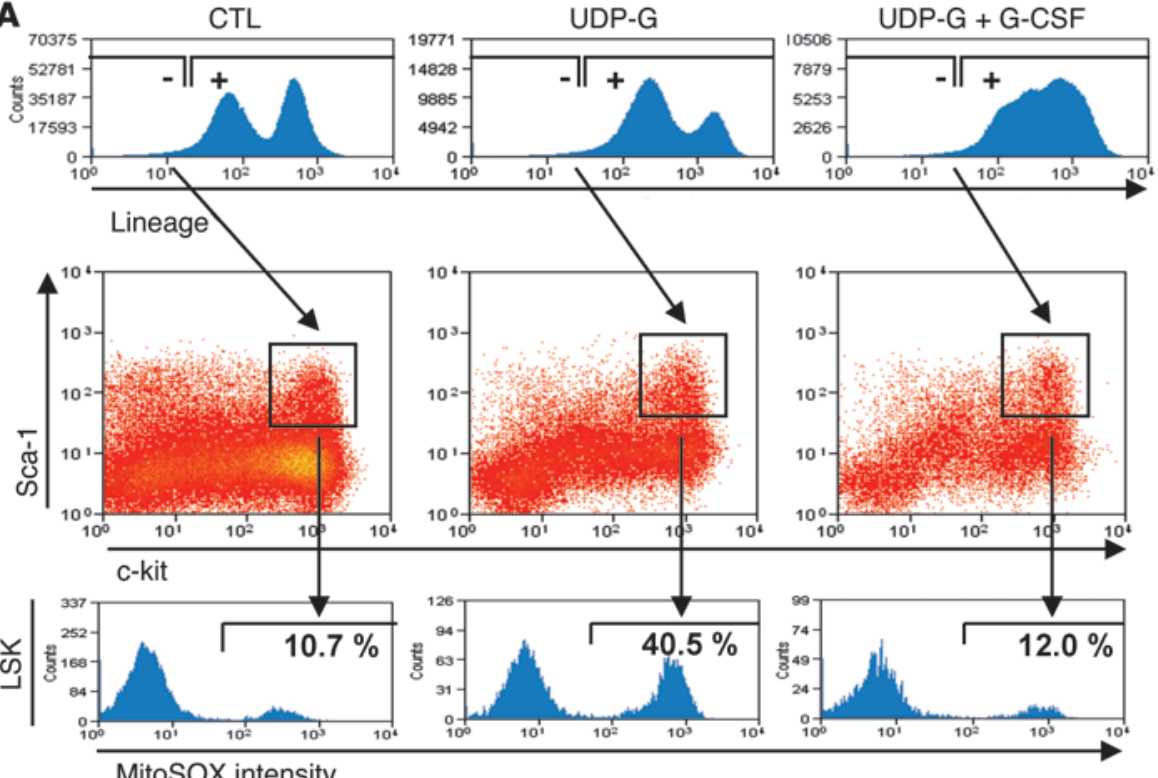

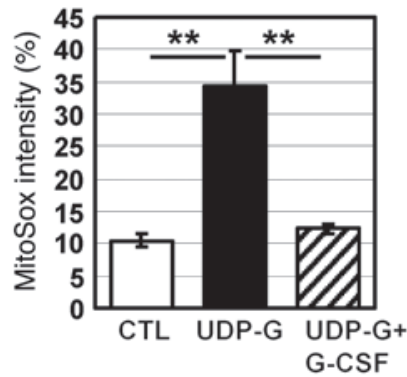

UDP-G

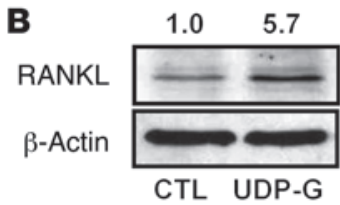

C

CTL
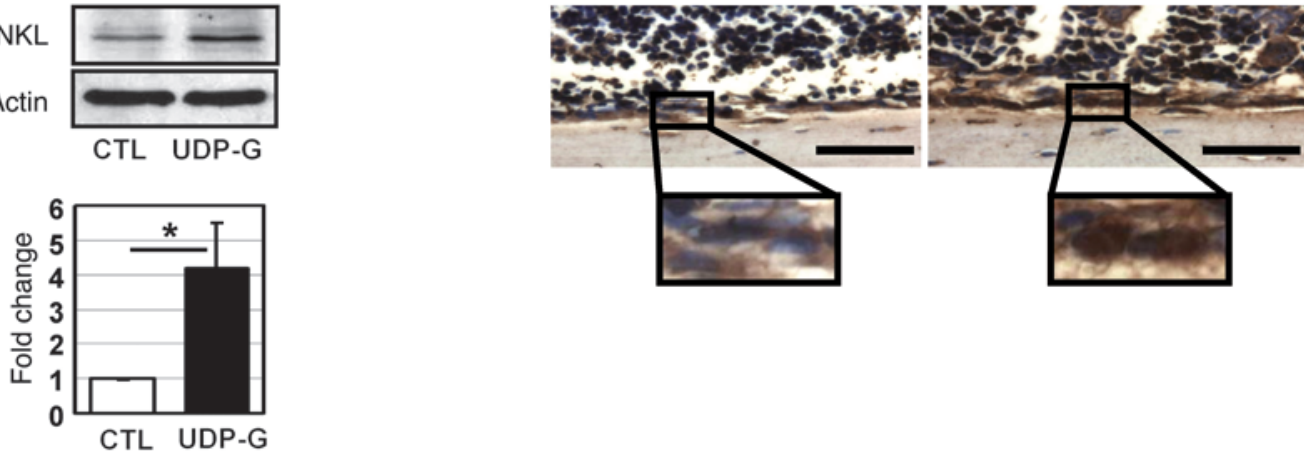

D

CTL

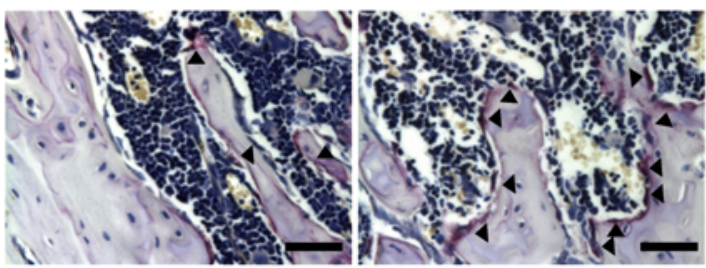

E

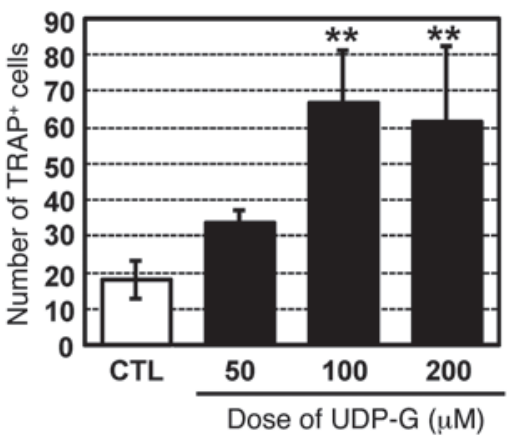

Figure 7

UDP-GIc increases mitochondrial ROS levels and promotes a transient osteoclast differentiation. (A) Mice were injected with UDP-GIc or UDP-GIc/G-CSF as described in Figure 6A. Lin- bone marrow cells (first row) were further gated (second row) to identify LSK cells. The cellular levels of mitochondrial superoxide were determined using MitoSOX-red within LSK cells (third row). Numbers indicate the percentage of gated cells. Data are representative of at least 4 mice analyzed individually per treatment group. (B) Mice were injected with UDP-Glc as described above. Bone marrow cell lysates were analyzed by Western blotting for RANKL expression. Values above each band represent fold difference in RANKL expression relative to control sample (vehicle injected) after normalization to $\beta$-actin loading control, as determined by densitometry. (C and D) Mice were treated as described above. (C) Femurs were sectioned longitudinally and immunostained with an antibody to RANKL. (D) Tissue sections were also stained for TRAP activity. Arrowheads indicate TRAP-positive cells. A representative TRAP staining is shown. Scale bar: $50 \mu \mathrm{M}$. (E) Bone marrow cells were pretreated with M-CSF and incubated with the indicated concentration of UDP-Glc. TRAP-positive cells were counted from at least 3 wells per treatment group. ${ }^{\star} P<0.05,{ }^{\star \star} P<0.01$. 
A
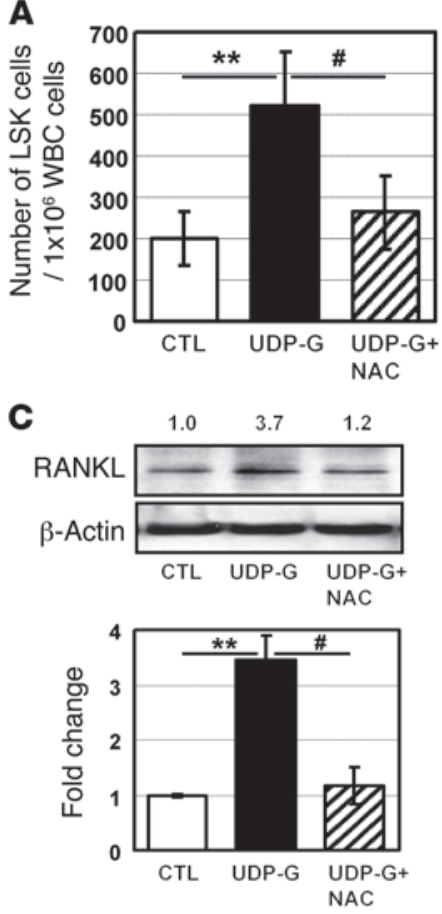

$\mathbf{E}$

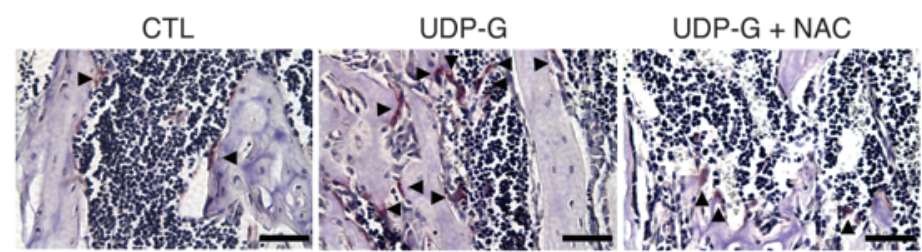

B

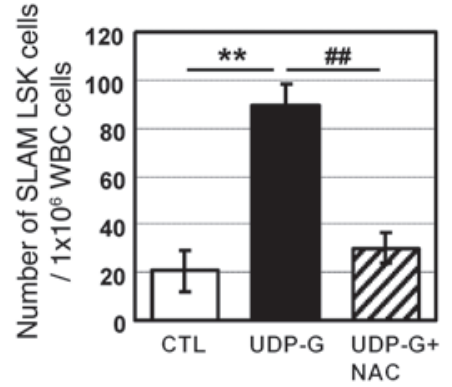

D

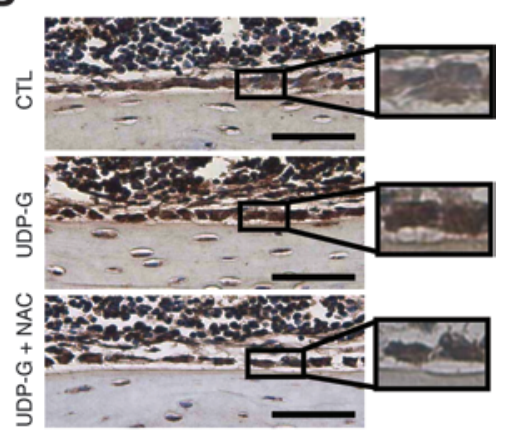

Figure 8

NAC abrogates UDP-Glc-induced mobilization. (A and B) Mice ( $n>4 /$ group) were treated with NAC as described in the Methods. UDP-GIC-mediated LSK (A) and SLAM LSK (B) cell mobilization was significantly suppressed by NAC treatment. The data shown are the mean \pm SD. (C and D) Mice were treated as described in A. RANKL expression was determined with Western blotting (C) and immunohistochemistry (D). In Western blot analysis, the numerical values represent the fold change in densitometry data (calculated as described above). Scale bar: $50 \mu \mathrm{M}$. (E) Mice were treated as indicated. Arrowheads indicate TRAP-positive cells. A representative TRAP staining is shown. Scale bar: $50 \mu \mathrm{M}$. ${ }^{\#} P<0.05,{ }^{\# \#} P<0.01,{ }^{* \star} P<0.01$.

a significantly higher portion of LSK and SLAM LSK cells in the recipient bone marrow were derived from UDP-Glc/G-CSF-treated mice (Figure 6, C and D).

UDP-Glc mobilizes HSPCs through the alterations of the osteoblast/osteoclast balance mediated by mitochondrial superoxide. It has been recently proposed that ROS signaling is closely associated with HSPC mobilization $(32,33)$. Thus, we examined whether UDP-Glc modulates the level of intracellular ROS levels in HSPCs. Since mitochondria are a major source of ROS, we measured the levels of mitochondrial superoxide in LSK cells. Upon UDP-Glc treatment, superoxide levels were significantly increased in LSK cells (Figure 7A). As increased intracellular ROS levels upregulate RANKL expression $(34,35)$, which could in turn enhance HSPC mobilization (36), we examined whether UDP-Glc has any direct effect on RANKL expression. UDP-Glc induced an increase of RANKL expression, as demonstrated by both West-

The combination of UDP-Glc with G-CSF improves HSPC mobilization. There is a keen interest in improving the mobilizing effects of G-CSF (11). Therefore, we investigated a possible combined effect of UDP-Glc and G-CSF. The mobilizing effect of UDP-Glc peaked 2-4 hours after the sixth daily consecutive injection (Figure 1), while G-CSF requires 4 consecutive daily injections (11). Based on these results, the administration schedule of the compounds was designed to synchronize the maximal effect of each treatment, as shown in Figure 6A. We first assessed the effects of these regimens by assessing the colony-forming activity. As shown in Figure 2, A and B, the combination of G-CSF with UDP-Glc produced significantly more CFU-Cs compared with G-CSF alone. CAFC activity was also highly enriched in UDPGlc/G-CSF-treated cells (Figure 2C), indicating that G-CSF, when combined with UDP-Glc, performed better in in vitro HPC assays. Similarly, the combination of UDP-Glc and G-CSF was more efficient in mobilizing LSK cells than either alone (Figure 2D). In the setting of competitive repopulation assay, UDP-Glc/ G-CSF-mobilized cells were dominant over G-CSF-mobilized cells throughout the whole posttransplantation period (Figure $6 \mathrm{~B})$. Although UDP-Glc alone was not as efficient as G-CSF in mobilizing in vitro colony-forming HPCs (Figure 2) or in vivo short-term repopulating cells (1 month posttransplantation in Figure 4A), a combination of UDP-Glc and G-CSF markedly enhanced short-term repopulating activity compared with G-CSF alone, and this competitive advantage continued for at least 5 months after transplantation (Figure 6B). Concomitantly, ern blot and immunohistochemical analyses (Figure 7, B and C). RANKL is a potent driver of osteoclast formation, and tipping the balance in favor of osteoclasts leads to mobilization of HSPCs (29). We observed a higher proportion of osteoclast cells in UDP-Glctreated mice, as evidenced by the expression of the osteoclast-associated enzyme tartrate-resistant acid phosphatase (TRAP) (Figure 7D). However, this UDP-Glc-induced osteoclastogenesis was transient, since the ratio of osteoblasts to osteoclasts returned to the prestimulation level 3-4 weeks after the treatment was stopped (Supplemental Figure 3). In an in vitro osteoclast differentiation assay, UDP-Glc also promoted the generation of osteoclasts (Figure 7E).

To investigate whether the elevated superoxide levels are indeed potential mediators of the UDP-Glc-mediated HSPC mobilization, the antioxidant $\mathrm{N}$-acetyl-L-cysteine (NAC) was administered. NAC was able to significantly abrogate the LSK and SLAM LSK cell mobilization induced by UDP-Glc (Figure 8, A and B), suggesting that superoxide acted as potential mediators in UDP-Glc mobilization. We then investigated whether the abrogation of UDPGlc mobilization by NAC occurs through inhibition of RANKL expression. Indeed, RANKL expression was notably reduced in NAC-treated animals in comparison with UDP-Glc-treated animals (Figure 8, C and D). Similarly, the level of UDP-Glc-induced osteoclastogenesis was significantly suppressed with NAC treatment (Figure 8E). Together, these findings suggest that UDP-Glc increases ROS levels, and this in turn enhances RANKL-induced osteoclast differentiation, leading to HSPC mobilization. Interestingly, while the combination of UDP-Glc and G-CSF augmented 
A

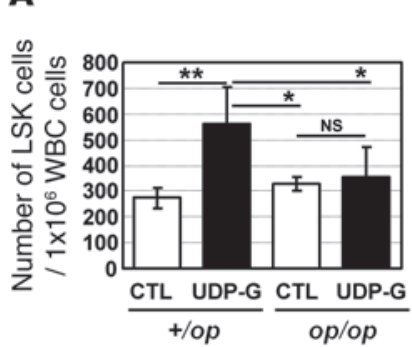

$\frac{\mathscr{D}}{\overline{\mathcal{D}}}$

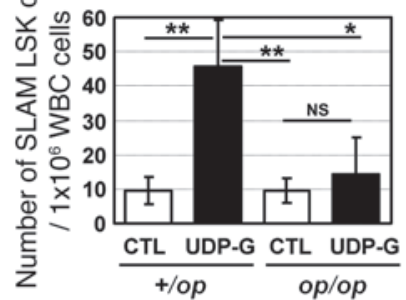

CTL

UDP-G

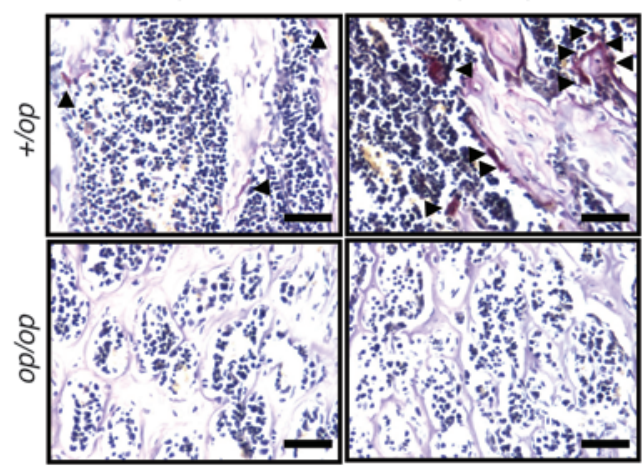

C
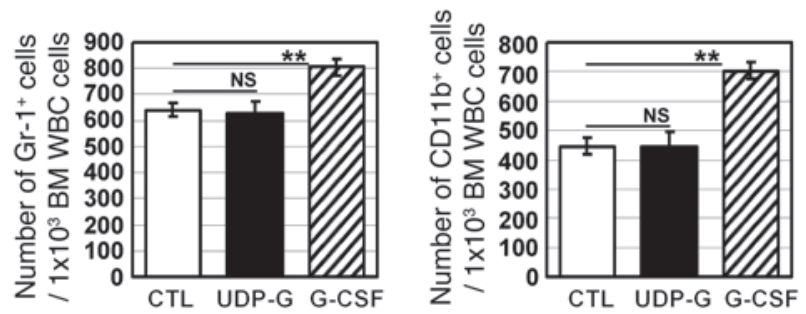

B
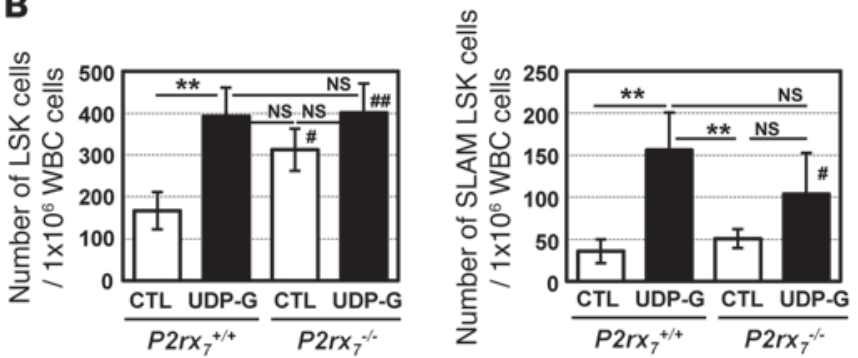

CTL

UDP-G

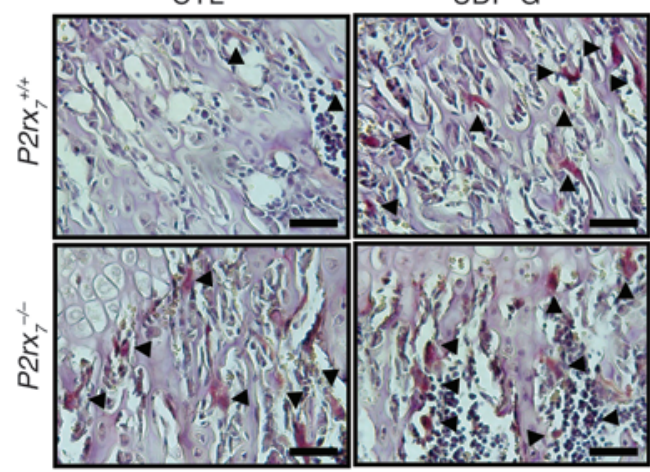

要

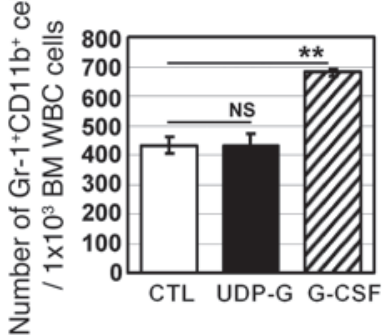

D

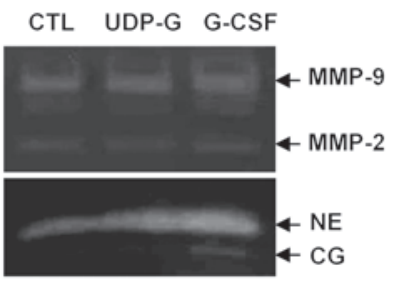

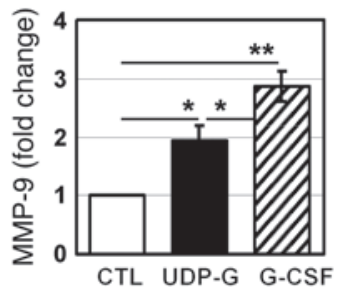

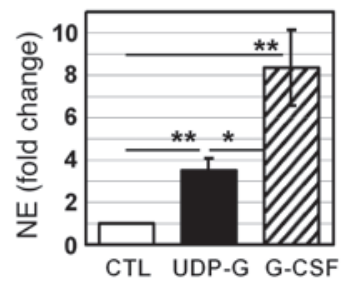

Figure 9

Role of osteoclasts in UDP-Glc-mediated HSPC mobilization. (A) op/op mutant mice $(n=6 /$ group) and littermate controls $(n=10 /$ group $)$ were treated with vehicle or UDP-GIc. HSPC mobilization was assessed by measuring the numbers of LSK and SLAM LSK cells in PB. In the representative images of TRAP staining, arrowheads indicate TRAP-positive cells. Scale bar: $50 \mu \mathrm{M}$. (B) P2rx $7^{-/-}$KO and WT controls $(n=10 /$ group $)$ were treated with vehicle or UDP-Glc. HSPC mobilization was assessed as described in A. In the representative images of TRAP staining, osteoclasts are present at high numbers at 6 weeks of age in P2rx $7^{--}$KO mouse. Data were obtained from 6- to 8-week-old animals. Arrowheads indicate TRAP-positive cells. Scale bar: $50 \mu \mathrm{M}$. (C) B6 mice were injected as described for Figure 4A. Bone marrow cells were collected from each treatment group and stained with indicated antibodies. Data represent 3 independent experiments. (D) Bone marrow mononuclear cells were isolated from B6 mice. To determine the protease activity, bone marrow supernatants were analyzed by zymogram analysis as described in the Methods. The intensity of the zymogram bands was analyzed utilizing densitometry, represented as fold change relative to vehicle-treated mice. Similar results were observed in 3 independent experiments. Data are mean $\pm \mathrm{SD} .{ }^{\star} P<0.05,{ }^{\star \star} P<0.01 ;{ }^{\#} P<0.05,{ }^{\# \#} P<0.01 \mathrm{vs}$. $P 2 \mathrm{rx} 7^{+/+} \mathrm{CTL}$.

HSPC mobilization, it significantly reduced mitochondrial superoxide levels compared with UDP-Glc alone (Figure 7A, right panel). This suggests that the combination of UDP-Glc and G-CSF augments the mobilizing effect through as yet unknown mechanisms, rather than via a further increment of ROS level.
Controversy still exists regarding the role of osteoclasts in regulating HSPC mobilization (36-39), raising the question of whether osteoclasts indeed play an essential role in UDP-Glc-mediated HSPC mobilization. To address this question, we first utilized the osteopetrotic $(o p / o p)$ mouse model. Mice homozygous for the op mutation exhibit 
A
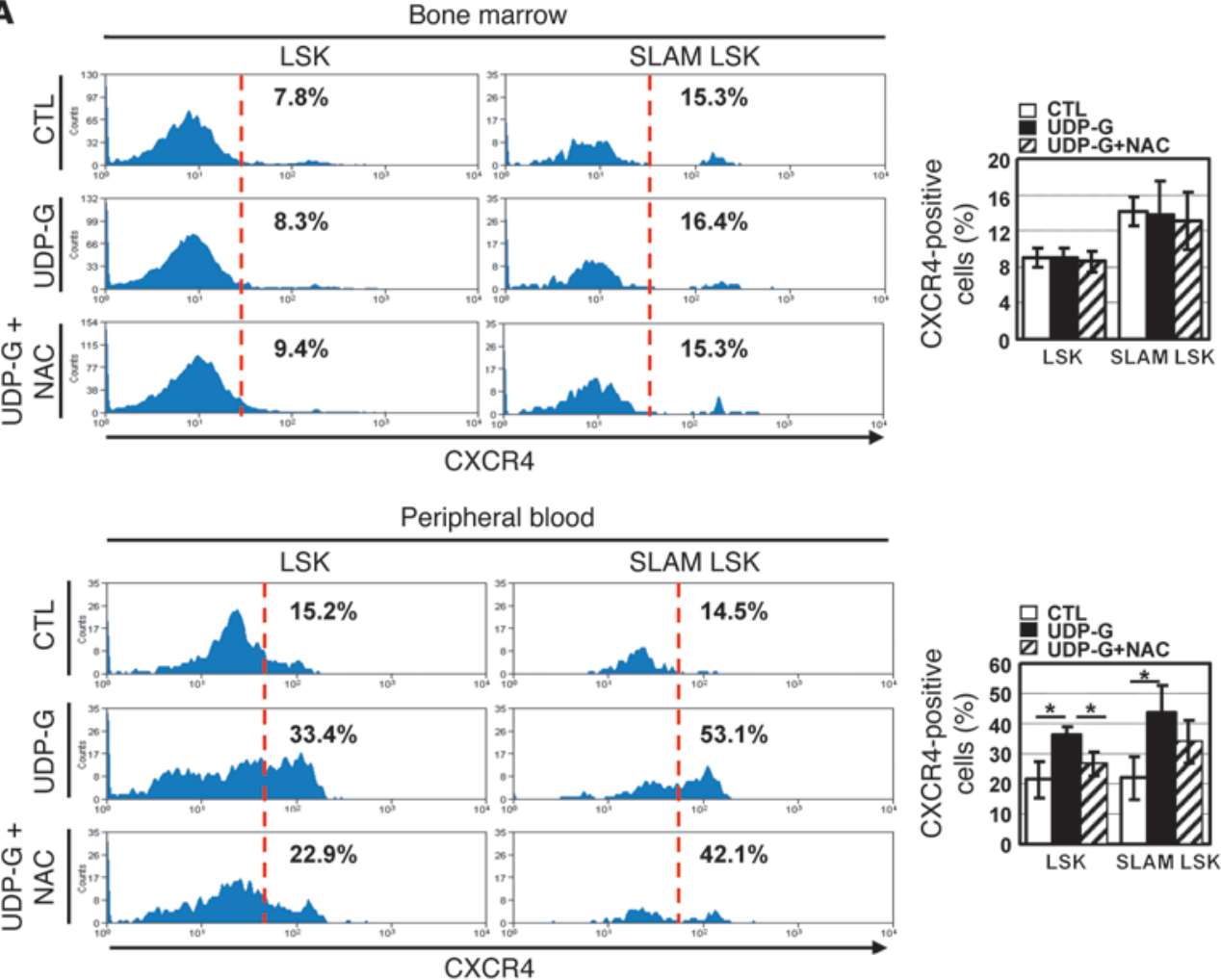

B
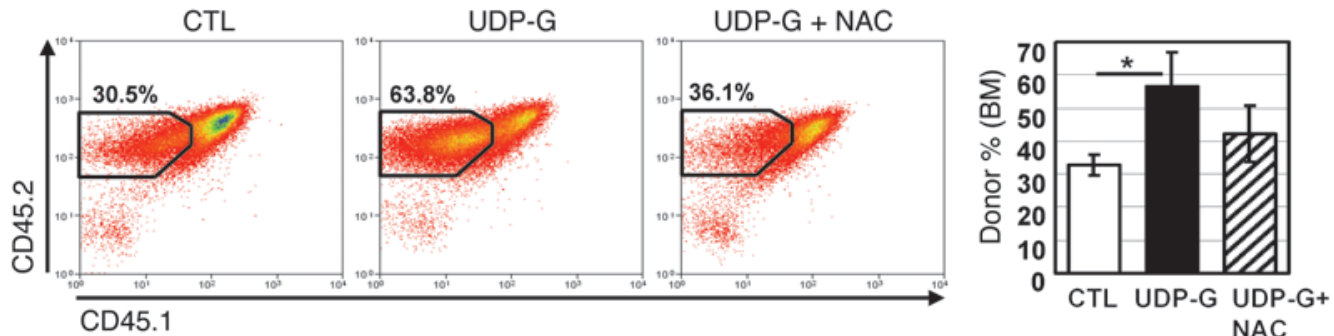

C

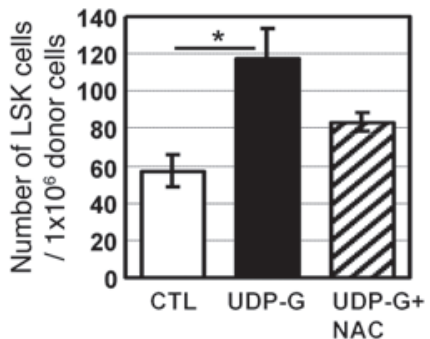

D

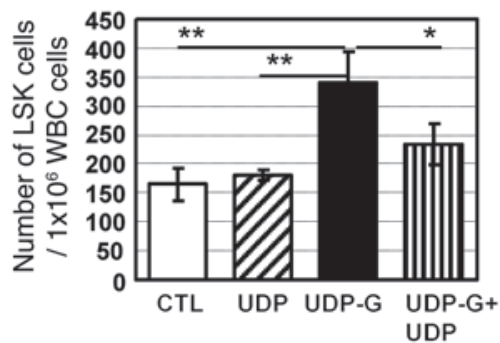

E

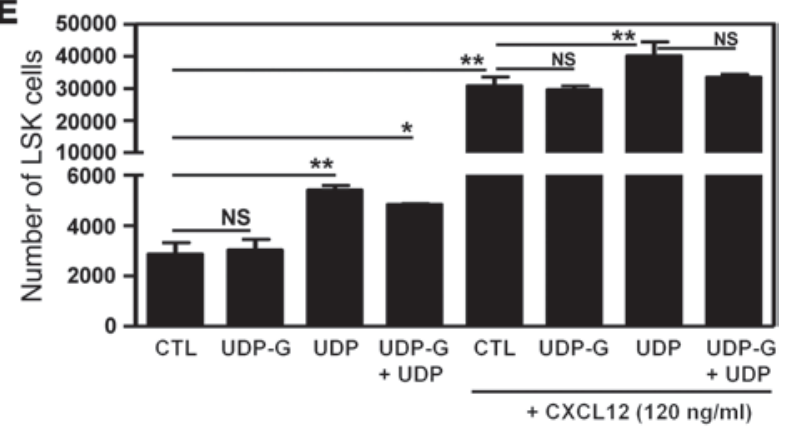

Figure 10

Analysis of HSPC migration in response to UDP and UDP-GIc. (A) Mice were treated as described in Figure 8. Expression levels of CXCR4 were determined in bone marrow and PB cells after gating on LSK or SLAM LSK subsets. Data are from at least 2 independent experiments with 4 mice/group. (B and C) B6 mice ( $n=7 /$ group) were treated with UDP-Glc or UDP-Glc/NAC as described in A. PB cells (CD45.2) were collected and injected into recipient mice (CD45.1.2) $\left(6 \times 10^{6} /\right.$ mouse), which were conditioned 24 hours before injection. Animals were sacrificed 12-14 hours after injection. (B) Recipient bone marrow cells were analyzed for expression of donor cell marker (CD45.2). (C) CD45.2+ cells were gated and analyzed for the presence of LSK cells. (D) B6 mice ( $n=5 / \mathrm{group}$ ) were injected with UDP (200 mg/kg), UDP-Glc (200 mg/kg), or UDP plus UDP-Glc and PB LSK cells quantified. (E) Chemotaxis assays were performed as described in Figure 1E. Lin- bone marrow cells were placed in the upper well (106/well); UDP, UDP-Glc, or UDP plus UDP-Glc (10 $\mu \mathrm{M}$ each) was placed in the lower wells. Cells that migrated to lower wells were collected and stained for Sca-1 and c-Kit. Data represent 2 independent experiments, each with duplicate wells per treatment condition. Data are mean \pm SD. ${ }^{*} P<0.05,{ }^{\star \star} P<0.01$. 
a severe deficiency of osteoclasts, and this strain can serve as a model to investigate the role of osteoclasts in UDP-Glc-mediated HSPC mobilization (40). Administration of UDP-Glc into littermate control mice $(\mathrm{CTL} ;+/ o p)$ induced osteoclastogenesis and promoted the mobilization of LSK cells and SLAM LSK cells (Figure 9A). However, op/op mice given the same treatment showed no changes in osteoclastogenesis and failed to show a statistically significant increase in the number of peripheral LSK and SLAM LSK cells (Figure 9A). These results suggest that osteoclasts play an important role in the regulation of UDP-Glc-mediated HSPC mobilization.

To further study the impact of osteoblasts/osteoclasts in UDPGlc-mediated HSPC mobilization, $\mathrm{P}_{2} \mathrm{X}_{7}$-deficient mice were analyzed. Deficiency of $\mathrm{P} 2 \mathrm{X}_{7}$ in the mouse results in impaired bone formation and excessive bone resorption (41). In accordance with this finding, a significantly increased numbers of osteoclasts were detected in untreated $\mathrm{P} 2 \mathrm{X}_{7} \mathrm{KO}$ mice (Figure 9B). UDP-Glc did not lead to a further noticeable increase in osteoclast activity in $\mathrm{P} 2 \mathrm{X}_{7}$ KO mice. Similarly, UDP-Glc-treated P2X 7 KO mice showed no significant increase in the number of peripheral LSK cells, compared with the vehicle-treated $\mathrm{P} 2 \mathrm{X}_{7} \mathrm{KO}$ mice (Figure 9B, top left). There was a trend toward moderately increased numbers of SLAM LSK cells ( 1.9-fold) in the blood of UDP-Glc-injected P2X 7 KO mice. However, this did not reach statistical significance. Notably, steady-state basal levels of circulating LSK cells were elevated in $\mathrm{P} 2 \mathrm{X}_{7} \mathrm{KO}$ mice compared with those in WT mice (Figure 9B, top left), suggesting the possibility that $\mathrm{P} 2 \mathrm{X}_{7}$ deficiency may lead to constitutive LSK cell mobilization in part through increased osteoclast activity. Taken together, these results suggest a potential role of osteoclasts in UDP-Glc-induced HSPC mobilization.

The proteolytic enzymes produced by monocytes/granulocytes are also important contributors mediating HSPCs mobilization (42). Thus, we examined whether UDP-Glc could induce the release of proteases from monocytes/granulocytes. As previously documented, there were overall increases in the percent of CD11 $\mathrm{b}^{+}$and/or Gr- $1^{+}$ cells in the bone marrow of G-CSF-treated mice; in contrast, UDPGlc did not lead to any notable changes in the percent of CD $11 \mathrm{~b}^{+}$ and/or Gr- $1^{+}$cells in the bone marrow (Figure 9C). Elevated levels of the proteolytic enzymes such as MMP-9, neutrophil elastase (NE), and cathepsin G (CG) were observed in mice treated with G-CSF (Figure 9D). UDP-Glc also elevated the level of MMP-9 and NE but at a significantly lower level compared with those treated with G-CSF (Figure 9D). There were no significant differences in MMP-2 and CG levels between vehicle- and UDP-Glc-injected groups.

We then determined the effect of UDP-Glc on the expression of CXCR4, which plays a key role in homing and mobilization of HSPCs (43). UDP-Glc-treated mice displayed no significant change in the percentage of CXCR4-expressing cells in their bone marrow LSK and SLAM LSK cells (Figure 10A, top panel). In contrast, UDP-Glc treatment led to an increased CXCR4 expression in the peripheral (circulating) LSK (33.4\% vs. $15.2 \%$ ) and SLAM LSK (53.1\% vs. $14.5 \%)$ populations (Figure 10A, bottom panel). NAC was able to abrogate the increase of CXCR4 expression induced by UDP-Glc, albeit not completely. When injected, UDP-Glc-mobilized PB cells homed to the bone marrow more efficiently than did vehicle-treated PB cells (Figure 10B). Homing of UDP-Glc-mobilized cells was inhibited by NAC pretreatment. A significantly higher number of donor-derived LSK cells $(P<0.05)$ was found in the bone marrow of mice transplanted with UDP-Glc-mobilized PB cells than in those of mice transplanted with vehicle-treated PB cells (Figure 10C). However, given that UDP-Glcmobilized PB contained greater numbers of LSK cells (Figure 2D), it is not clear whether UDP-Glc has a major effect on the homing capacity of the mobilized LSK cells through its upregulation of CXCR4.

It was previously shown that UDP antagonizes the action of UDP-Glc (44). We therefore evaluated the effects of UDP on UDPGlc-mediated HSPCs migration. UDP alone did not elicit any appreciable change in the number of LSK cells in the peripheral circulation (Figure 10D). However, when UDP was simultaneously treated with UDP-Glc, it antagonized UDP-Glc-induced LSK cell mobilization (Figure 10D). Interestingly, UDP could chemoattract LSK cells in vitro in the presence or absence of CXCL12 (Figure $10 \mathrm{E})$. Conversely, UDP-Glc treatment led to a moderate inhibition of UDP-induced chemotaxis, but this did not reach the statistical significance in the presence of CXCL12.

\section{Discussion}

Mobilized HSPCs could regenerate a complete hematopoietic system for cancer patients with hematolymphoid malignancies or solid tumors. Yet more than $20 \%$ of patients fail to mobilize sufficient stem cells for transplantation (45). These include patients who were previously treated with intensive radiation and chemotherapy, those who have genetic disorders such as FA, and those who are over 60 years of age $(11,46)$. A combination of G-CSF with cytotoxic agents improves HSPC mobilization in poor-mobilizer patients, but this is often accompanied by serious side effects (47). Such limitations necessitate the discovery of novel mobilizing regimens, so that therapy can be tailored to the individual. The data presented in the current study establish what we believe is a novel aspect of the nucleotide sugar, UDP-Glc, in HSPC mobilization.

Functional characteristics of HSPCs, such as homing, engraftment, cell cycle status, and self-renewal activity vary according to their tissue of origin $(14,48)$. For example, circulating blood stem cells can not compete effectively against bone marrow-derived stem cells for long-term multilineage repopulation (49). Therefore, when mobilized cells are assessed for their functional activity, it is more legitimate to compare cells from the same tissue origin, i.e., G-CSF-mobilized PB versus UDP-Glc-mobilized PB. To this end, we adapted a competitive repopulation assay in which a mixture of equal numbers of UDP-Glc- and G-CSF-mobilized blood cells were transplanted into conditioned recipients (see Supplemental Figure 2), which allowed a direct comparison of UDP-Glc-mobilized cells with G-CSF-mobilized cells under the same microenvironment.

Following administration of UDP-Glc, the spleen and blood contained increased numbers of CFU-Cs. Since the frequency of HSPCs in the spleen is about $10 \%$ of that of bone marrow, the increase of CFU-Cs in the spleen (Figure 1B and Figure 2B) may have been the result of mobilization, expansion of resident splenic HSPCs, or (most likely) both. While UDP-Glc-mobilized cells had a lower capacity to form in vitro colonies compared with G-CSFmobilized cells, serial transplantation experiments showed that UDP-Glc preferentially mobilized long-term self-renewing HSPCs. UDP-Glc-mobilized PB contained a greater number of SLAM LSK cells than G-CSF-mobilized cells, which could provide a rationale for their superior long-term repopulating ability. However, when sorted peripheral SLAM LSK cells from UDP-Glc mobilization were compared with equal numbers of counterpart cells from G-CSF mobilization in competitive serial transplantation models (Figure 4G), UDP-Glc-mobilized SLAM LSK cells consistently displayed superior long-term repopulating ability, suggesting that the qualitative advantage of UDP-Glc-mobilized HSPCs also account for the superior engraftment potential of UDP-Glc-mobilized cells. 
Cytokine-induced stem cell mobilization is often accompanied by profound changes in number and composition of accessory cells contained within the PBSC collection (50). In contrast, UDP-Glc did not cause any noticeable quantitative changes in the accessory cell compartment (Figure 3A). Accessory cells, especially $T$ cells, exert immune regulatory function and influence the development of GVHD and graft-versus-leukemia (GVL) in the allogeneic setting (51). UDP-Glc appears to have minimal effects on peripheral $\mathrm{T}$ cell numbers, which may be beneficial in reducing the incidence and severity of GVHD in the setting of certain clinical situations. When transplanted, the skewing of the lymphoid/ myeloid ratio toward the lymphoid lineage was pronounced in the UDP-Glc-mobilized HSPCs (Figure 5, B and C). In addition, UDPGlc did not disrupt cell cycle quiescence of HSPCs, and this could contribute to the enhanced long-term engraftment potential of UDP-Glc-mobilized cells. All of these findings indicate that UDPGlc is a previously unrecognized HSPC mobilizer that egresses a functionally distinct subset of the HSPCs.

Redox signaling plays a central role in regulating HSPC mobilization (32), because many of the cytokines, chemokines, and adhesion molecules associated with HSPC mobilization are regulated through a redox-regulated process (52). Mice treated with UDPGlc expressed high levels of mitochondrial superoxide in their HSPCs. Using antioxidants to lower these mitochondrial superoxide levels significantly reduced the mobilizing effect of UDPGlc, and this coincided with the reduction in RANKL and osteoclastogenesis (Figure 8). These results therefore suggest that ROS play a role in mediating the UDP-Glc-induced HSPC mobilization through an increase of RANKL expression and osteoclast activity.

It has been shown that osteoclasts mediate HSPC egress from the endosteal osteoblastic niche by degrading endosteal components $(36,37,39)$. However, other lines of evidence indicate that osteoclasts are dispensable for HSC mobilization (38). UDP-Glc mobilization was not achievable in mouse models of osteopetrosis (op/op), suggesting that osteoclast formation is required for UDP-Glcmediated HSPC mobilization. On the other hand, $\mathrm{P} 2 \mathrm{X}_{7} \mathrm{KO}$ mice, which display excessive osteoclast resorption activity at 6-8 weeks of age (41), showed a higher number of circulating LSK cells than WT mice, even under steady-state conditions. UDP-Glc did not further increase osteoclast formation in $\mathrm{P} 2 \mathrm{X}_{7} \mathrm{KO}$ mice that were already osteoporotic, and no significant change was observed in the number of peripheral LSK cells in UDP-Glc-injected P2 $\mathrm{X}_{7} \mathrm{KO}$ mice. Taken together, our findings suggest that the extent of osteoclast formation in response to UDP-Glc is functionally associated with the ability of UDP-Glc to mobilize HSPCs.

Other mechanisms for UDP-Glc-induced mobilization would be an indirect effect involving activation of neutrophils with the subsequent release of proteases (53): increased levels of proteases can attack several target proteins, including CXCR4, CXCL12 (SDF$1 \alpha$ ), or VCAM-1, leading to inactivation of CXCR4/CXCL12- or VCAM-1/VLA-4-dependent signals and thus cell migration out of bone marrow. Unlike G-CSF, however, UDP-Glc had no effect on granulocyte and monocyte mobilization (Figure 3A). Furthermore, UDP-Glc was able to mobilize HSPCs in G-CSFR-deficient ( $\mathrm{Cs} f 3 \mathrm{r}^{-/}$) mice, although to a lesser extent than in WT mice (Figure 3B), suggesting that granulocyte and monocyte mobilization does not appear to be essential in UDP-Glc-mediated mobilization. Despite the insignificant impact on granulocytes and monocytes, there were trends toward modest increases in the levels of MMP-9 and NE (but not MMP-2 and CG) in the bone marrow of UDP-Glc-injected mice
(Figure 9D). While this increase was substantially lower in magnitude compared with increases in G-CSF-treated mice, we cannot exclude the possibility that proteolytic enzymes contribute, at least to some extent, to UDP-Glc-mediated mobilization.

UDP-Glc treatment increased CXCR4 cell surface expression in peripheral HSPCs but not in bone marrow HSPCs (Figure $10 \mathrm{~A})$. However, it remains an open question as to whether or not the increased CXCR4 expression by UDP-Glc is associated with increased homing capacity.

UDP-Glc is known to bind the P2RY14 receptor. It is therefore of interest to investigate whether UDP-Glc triggers HSPC mobilization through P2RY14 receptor-dependent or -independent mechanisms (or both). While this area warrants further study using animal models such as conditional P2ry14 KO animals, there are contradictory reports that UDP-Glc is not a functionally relevant ligand at P2RY14 receptor $(54,55)$. It is also noteworthy that HSPC mobilization is often mediated through multiple trans-acting signals rather than ligand-receptor interactions $(56,57)$. Recent studies established the biological significance of extracellular nucleotides in migration and engraftment of human HSPCs $(19,20)$ : UTP has the capacity to chemoattract human $\mathrm{CD} 34^{+}$cells and enhances engraftment of human HSPCs. Because chemokine/chemokine receptor axes play a critical role in HSPC mobilization, it is notable that P2RY14 also encodes a 7-transmembrane G-protein coupled receptor (GPCR) with a chemokine receptor signature (e.g., DRY motif in the 3 rd intracellular domain) (58). However, UDP-Glc did not display chemoattractive properties on mouse LSK cells. Rather, UDP, which antagonizes the action of UDP-Glc (44), had chemotactic activity and attracted mouse LSK cells (Figure 10).

Quiescent HSCs have higher long-term repopulating abilities than do HSCs in active cell cycle (59). Since UDP-Glc does not affect cell cycle quiescence of bone marrow-resident HSPCs (Figure 5A), this could contribute to the enhanced long-term engraftment potential of UDP-Glc-mobilized cells. How UDPGlc mobilizes HSPCs without disrupting cell cycle quiescence remains unknown. Unlike cytotoxic drug- and/or cytokine-based mobilizations that are often accompanied by marked changes in cell proliferation, cell death, and bone marrow sinusoidal endothelium, UDP-Glc did not lead to significant changes in any of these secondary events. It is also conceivable, although still speculative, that UDP-Glc may regulate cell cycle progression through modulation of cyclin-dependent kinase inhibitors.

UDP-Glc is a naturally occurring metabolite in the human body, so that it may mitigate many of the side effects often associated with other synthetic mobilizers. Indeed, none of the UDP-Glc-treated animals showed signs of side effects such as spleen enlargement (Supplemental Figure 4). They appeared normal and healthy during the course of the study. Furthermore, UDP-Glc-induced osteoclastogenesis is only temporary (reversible) (Supplemental Figure 3). Nevertheless, when a new compound is considered for clinical development, it is generally recommended to initiate therapy at a low dose to minimize potential adverse effects. A high-potency UDPGlc, MRS 2690, displays approximately 7-fold higher potency than UDP-Glc (60), and became commercially available in recent years. This new compound may allow a reduction in the dose of UDP-Glc needed while maintaining the desired effects.

Considering the high cost, side effects, and ineffectiveness of conventional mobilization regimens, there is a compelling need to seek alternative mobilization regimens. UDP-Glc mobilizes 
functionally distinct subsets of HSPCs compared with those mobilized by G-CSF, suggesting the possibility that the combination regimen might enhance both short- and long-term repopulating capacity of the mobilized cells. In this context, UDP-Glc can be utilized as a complement regimen that potentiates the long-term repopulating capacity of G-CSF-mobilized HSPCs. Therefore, on the basis of our observations, UDP-Glc mobilization, either alone or combined with G-CSF, could potentially provide a scientific basis for improving transplantation outcomes. Moreover, UDP-Glc minimally affects the immune cell content of the mobilized cells, and this may alter the likelihoods of graft failure, GVHD, and GVL.

The small size of UDP-Glc offers other tangible advantages over other protein-based mobilizers, including easy access to intracellular targets, low cost, and ease of production as well as oral bioavailability. Administration of UDP-Glc appeared to be well tolerated at high doses, suggesting the potential suitability as a therapeutic agent in humans. Clearly, further pharmacokinetic data will be required in pursuit of more effective HSPC mobilization and to lessen toxicity to donors and recipients. Our results may have important clinical implications in designing new mobilization strategies to improve the efficiency and outcome of autologous and allogeneic PBSC transplantation.

\section{Methods}

Animals and treatment. Mice received s.c. injections of UDP-Glc $(200 \mathrm{mg} / \mathrm{kg}$; Sigma-Aldrich) dissolved in sterile endotoxin-free PBS. G-CSF (Neupogen; Amgen) was administered daily at a dose of $300 \mu \mathrm{g} / \mathrm{kg}$ s.c. for 4 consecutive days as previously described (11). For the combination group, mice were injected UDP-Glc at $200 \mathrm{mg} / \mathrm{kg}$ s.c. for 6 consecutive days (from day 0 to day 5), accompanied by $300 \mu \mathrm{g} / \mathrm{kg}$ s.c. injections of G-CSF (from day 2 to day 5). NAC (Sigma-Aldrich) was administered s.c. at $100 \mathrm{mg} / \mathrm{kg} /$ day.

CFU-C and CAFC. Mobilized mononuclear PB cells and spleen cells were seeded for the colony-forming cell (CFC) assay. The numbers of BFU-E, CFU-GM, and CFU-GEMM colonies were counted using standard criteria. CAFC was performed as described previously (61).

Transplantation. For competitive repopulation assays, an equal number of PB cells mobilized by each agent (PBS vs. UDP-Glc, UDP-Glc vs. G-CSF, and G-CSF vs. UDP-Glc/G-CSF) were transplanted into conditioned recipient mice (CD45.1.2., 9.5-10 Gy). Although we used CD45 congenic animals (B6) in competitive repopulation assay, in order to confirm that our results are not due to potential variability resulting from the disparity between the CD45.1 and CD45.2, the results were further confirmed by reciprocal injections of mobilizers (i.e., inject G-CSF into CD45.1 and UDP-Glc to CD45.2 mice, and vice versa). The ratio of CD45.1/CD45.2 cells in recipient PB was determined at various times after transplantation. When we transplant sorted CD $45.1^{+}$SLAM LSK or CD 45.2 $2^{+}$SLAM LSK cells into lethally irradiated animals, $1-2 \times 10^{6} \mathrm{~PB}$ cells (CD45.1.2) were co-administered whose contribution to recipient hematopoietic reconstitution is minimal.

Flow cytometry analysis. The relative contributions of UDP-Glc-, G-CSF-, and UDP-Glc/G-CSF-mobilized PB cells to recipient blood and bone marrow were assessed by flow cytometry analysis using anti-CD45.1 and anti-CD45.2 antibodies (eBioscience). PB LSK and SLAM LSK cells were phenotyped using the following antibodies: lineage markers PE-Cy7-conjugated anti-CD3, anti-CD4, anti-CD8, anti-CD45R, anti-CD11b, antiGr-1, and anti-TER-119 (eBioscience); PE-conjugated anti-Sca-1 (eBioscience); APC-conjugated anti-c-Kit (eBioscience); perCP/Cy5.5-conjuated anti-CD150 (BioLegend); Pacific Blue-conjugated anti-CD48 (BioLegend). The percentage of bone marrow LSK and SLAM LSK cells derived from UDP-Glc- and G-CSF-mobilized cells was analyzed among the gated CD45.1 or CD45.2 compartment. Mitochondrial superoxide level was measured using MitoSox (Invitrogen) within LSK cells according to the manufacturer's instructions.

TRAP staining and immunohistochemistry. Femurs dissected from treated mice were embedded in paraffin and longitudinally cut to $5-\mu \mathrm{m}$ thickness. For identification of osteoclasts, the sections were stained using a TRAP staining kit (B-Bridge International Inc.) according to the manufacturer's instructions. For the in vitro osteoclast differentiation assay, bone marrow cells $\left(2 \times 10^{5}\right)$ were pretreated with $20 \mathrm{ng} / \mathrm{ml} \mathrm{M}$-CSF (eBioscience) for 3 days and further cultured for 4 days with various concentrations (0-200 $\mu \mathrm{M})$ of UDPGlc. After 7 days of incubation, cells were stained and counted as described above. For immunohistochemical staining of RANKL, the sections were incubated overnight with anti-mouse RANKL (Santa Cruz Biotechnology; 1:50).

Chemotaxis assay. For chemotactic assays, lineage-depleted cells $\left(10^{6} /\right.$ well) were placed in the upper chamber. UDP, UTP, and UDP-Glc $(10 \mu \mathrm{M}$; Sigma-Aldrich) were placed to the bottom chamber with or without CXCL12 (120 ng/ml; Peprotech). After 6 hours of incubation, migrated cells were stained with FITC-conjugated anti-mouse Sca-1 (eBioscience) and APC-conjugated anti-mouse c-Kit (eBioscience). Flow cytometry was used to enumerate migrated cells.

Zymographic analysis. For zymography, bone marrow supernatants were loaded on $10 \%$ precasted polyacrylamide gel with gelatin for MMP-2 and MMP-9, and 12.5\% precasted polyacrylamide gel (Bio-Rad) with casein for NE and CG under non-reducing conditions. After electrophoresis, the gels were washed in zymogram renaturation buffer (2.5\% Triton X-100) and then incubated overnight at $37^{\circ} \mathrm{C}$ in zymogram development buffer (Bio-Rad). The gels were then stained with $0.5 \%$ Coomassie blue solution and destained. Proteinase activity was determined using colorless zones against a blue background.

Statistics. All data are expressed as mean \pm SD. One-way ANOVA was used for multiple comparisons using SPSS version 16.0 software. $P<0.05$ was considered statistically significant.

Study approval. All animal studies were conducted after review and approval by the University of Pittsburgh's Institutional Animal Care and Use Committee and in accordance with the University of Pittsburgh's Policy on the Care, Welfare, and Treatment of Laboratory Animals.

\section{Acknowledgments}

The authors wish to thank Sid Kar and James Kim for scientific editing of the manuscript and Tsvee Lapidot, Orit Kollet, Eduardo Lazarowski, and Markus Mapara for the helpful discussions and suggestions. This study was supported in part by research funding from Department of Defense (grant W81XWH-09-1-0364) to B.C. Lee. B.C. Lee is a recipient of the Junior Scholar Award from the Department of Medicine, University of Pittsburgh School of Medicine. This project used the UPCI flow cytometry and animal facility, which were supported in part by the P30CA047904 award from the NIH.

Received for publication March 30, 2012, and accepted in revised form May 16, 2013.

Address correspondence to: Byeong-Chel Lee, University of Pittsburgh Cancer Institute, Hillman Cancer Center, 5117 Centre Avenue, Pittsburgh, Pennsylvania 15213, USA. Phone: 412.623.2285; Fax: 412.623.7828; E-mail: leeb4@upmc.edu.

Sungho Kook's present address is: Institute of Oral Biosciences and School of Dentistry, Chonbuk National University, Jeonju, South Korea. 
1. Hartmann O, et al. Peripheral blood stem cell and bone marrow transplantation for solid tumors and lymphomas: hematologic recovery and costs. A randomized, controlled trial. Ann Intern Med. 1997;126(8):600-607.

2. Tricot $G$, et al. Peripheral blood stem cell transplants for multiple myeloma: identification of favorable variables for rapid engraftment in 225 patients. Blood. 1995;85(2):588-596

3. Neben S, Marcus K, Mauch P. Mobilization of hematopoietic stem and progenitor cell subpopulations from the marrow to the blood of mice following cyclophosphamide and/or granulocyte colony-stimulating factor. Blood. 1993;81(7):1960-1967.

4. Majolino I, Aversa F, Bacigalupo A, Bandini G, Arcese W, Reali G. Allogeneic transplants of rhG-CSF-mobilized peripheral blood stem cells (PBSC) from normal donors. GITMO. Gruppo Italiano Trapianto di Midollo Osseo. Haematologica. 1995;80(1):40-43.

5. Platzbecker U, et al. Spleen enlargement in healthy donors during G-CSF mobilization of PBPCs. Transfusion. 2001;41(2):184-189.

6. Yeoh JS, Ausema A, Wierenga P, de Haan G, van Os R. Mobilized peripheral blood stem cells provide rapid reconstitution but impaired long-term engraftment in a mouse model. Bone Marrow Transplant. 2007;39(7):401-409.

7. Borgerding JN, et al. G-CSF treatment induces hematopoietic stem cell quiescence but loss of repopulating activity. Presented at: American Society of Hematology 54th Annual Meeting December 2012; Atlanta, Georgia, USA.

8. Hill GR, et al. Stem cell mobilization with G-CSF induces type 17 differentiation and promotes scleroderma. Blood. 2010;116(5):819-828.

9. Pulliam AC, et al. AMD3100 synergizes with G-CSF to mobilize repopulating stem cells in Fanconi anemia knockout mice. Exp Hematol. 2008;36(9):1084-1090.

10. D'Addio A, et al. The addition of plerixafor is safe and allows adequate PBSC collection in multiple myeloma and lymphoma patients poor mobilizers after chemotherapy and G-CSF. Bone Marrow Transplant. 2011;46(3):356-363.

11. Broxmeyer HE, et al. Rapid mobilization of murine and human hematopoietic stem and progenitor cells with AMD3100, a CXCR4 antagonist. J Exp Med. 2005;201(8):1307-1318.

12. Wright DE, Cheshier SH, Wagers AJ, Randall TD, Christensen JL, Weissman IL. Cyclophosphamide/ granulocyte colony-stimulating factor causes selective mobilization of bone marrow hematopoietic stem cells into the blood after $M$ phase of the cell cycle. Blood. 2001;97(8):2278-2285.

13. Mollee P, et al. Cyclophosphamide, etoposide and G-CSF to mobilize peripheral blood stem cells for autologous stem cell transplantation in patients with lymphoma. Bone Marrow Transplant. 2002;30(5):273-278.

14. Lapid K, Vagima Y, Kollet O, Lapidot T. Egress and mobilization of hematopoietic stem and progenitor cells. In: StemBook. Cambridge, Massachusetts, USA: Harvard Stem Cell Institute; 2008.

15. Di Virgilio F, et al. Nucleotide receptors: an emerging family of regulatory molecules in blood cells. Blood. 2001;97(3):587-600.

16. Sak K, Boeynaems JM, Everaus H. Involvement of $\mathrm{P} 2 \mathrm{Y}$ receptors in the differentiation of haematopoietic cells. J Lenkoc Biol. 2003;73(4):442-447.

17. Linden J. Cell biology. Purinergic chemotaxis. Science. 2006;314(5806):1689-1690.

18. Chen Y, et al. ATP release guides neutrophil chemotaxis via P2Y2 and A3 receptors. Science. 2006;314(5806):1792-1795

19. Rossi L, et al. The extracellular nucleotide UTP is a potent inducer of hematopoietic stem cell migration. Blood. 2007;109(2):533-542.

20. Lemoli RM, et al. Extracellular nucleotides are potent stimulators of human hematopoietic stem cells in vitro and in vivo. Blood. 2004;104(6):1662-1670.
21. Abbracchio MP, Burnstock G. Purinergic signalling: pathophysiological roles. Jpn J Pharmacol. 1998;78(2):113-145.

22. Lazarowski ER, Shea DA, Boucher RC, Harden TK. Release of cellular UDP-glucose as a potential extracellular signaling molecule. Mol Pharmacol. 2003;63(5):1190-1197.

23. Arase T, et al. The UDP-glucose receptor P2RY14 triggers innate mucosal immunity in the female reproductive tract by inducing IL-8. J Immunol. 2009;182(11):7074-7084.

24. Eigenbrodt E, Reinacher M, Scheefers-Borchel U, Scheefers H, Friis R. Double role for pyruvate kinase type $\mathrm{M} 2$ in the expansion of phosphometabolite pools found in tumor cells. Crit Rev Oncog. 1992;3(1-2):91-115.

25. He S, Kim I, Lim MS, Morrison SJ. Sox17 expression confers self-renewal potential and fetal stem cell characteristics upon adult hematopoietic progenitors. Genes Dev. 2011;25(15):1613-1627.

26. Roberts AW, Foote S, Alexander WS, Scott C, Robb L, Metcalf D. Genetic influences determining progenitor cell mobilization and leukocytosis induced by granulocyte colony-stimulating factor. Blood. 1997;89(8):2736-2744.

27. Park CY, Majeti R, Weissman IL. In vivo evaluation of human hematopoiesis through xenotransplantation of purified hematopoietic stem cells from umbilical cord blood. Nat Protoc. 2008;3(12):1932-1940.

28. de Kruijf EJ, Hagoort H, Velders GA, Fibbe WE, van Pel M. Hematopoietic stem and progenitor cells are differentially mobilized depending on the duration of Flt3-ligand administration. Haematologica. 2010;95(7):1061-1067.

29. Purton LE, Scadden DT. Osteoclasts eat stem cells out of house and home. Nat Med. 2006;12(6):610-611.

30. Liu F, Wu HY, Wesselschmidt R, Kornaga T, Link DC. Impaired production and increased apoptosis of neutrophils in granulocyte colony-stimulating factor receptor-deficient mice. Immunity. 1996;5(5):491-501.

31. Steinman RA. Cell cycle regulators and hematopoiesis. Oncogene. 2002;21(21):3403-3413.

32. Tesio M, et al. Enhanced c-Met activity promotes G-CSF-induced mobilization of hematopoietic progenitor cells via ROS signaling. Blood. 2011;117(2):419-428.

33. Dar A, et al. Rapid mobilization of hematopoietic progenitors by AMD3100 and catecholamines is mediated by CXCR4-dependent SDF-1 release from bone marrow stromal cells. Lenkemia. 2011;25(8):1286-1296.

34. Bai XC, et al. Reactive oxygen species stimulates receptor activator of NF-kappaB ligand expression in osteoblast. J Biol Chem. 2005;280(17):17497-17506.

35. Barsony J, Sugimura Y, Verbalis JG. Osteoclast response to low extracellular sodium and the mechanism of hyponatremia-induced bone loss. J Biol Chem. 2011;286(12):10864-10875.

36 . Kollet $\mathrm{O}$, et al. Osteoclasts degrade endosteal components and promote mobilization of hematopoietic progenitor cells. Nat Med. 2006;12(6):657-664.

37. Calvi LM, et al. Osteoblastic cells regulate the haematopoietic stem cell niche. Nature. 2003;425(6960):841-846.

38. Miyamoto K, et al. Osteoclasts are dispensable for hematopoietic stem cell maintenance and mobilization. J Exp Med. 2011;208(11):2175-2181.

39. Takamatsu Y, Simmons PJ, Moore RJ, Morris HA, To LB, Levesque JP. Osteoclast-mediated bone resorption is stimulated during short-term administration of granulocyte colony-stimulating factor but is not responsible for hematopoietic progenitor cell mobilization. Blood. 1998;92(9):3465-3473.

40. Yoshida H, et al. The murine mutation osteopetrosis is in the coding region of the macrophage colony stimulating factor gene. Nature. 1990;345(6274):442-444.

41. Ke HZ, et al. Deletion of the P2X7 nucleotide receptor reveals its regulatory roles in bone formation and resorption. Mol Endocrinol. 2003;17(7):1356-1367.
42. Levesque JP, Hendy J, Takamatsu Y, Williams B, Winkler IG, Simmons PJ. Mobilization by either cyclophosphamide or granulocyte colony-stimulating factor transforms the bone marrow into a highly proteolytic environment. Exp Hematol. 2002;30(5):440-449.

43. Peled A, et al. Dependence of human stem cell engraftment and repopulation of NOD/SCID mice on CXCR4. Science. 1999;283(5403):845-848.

44. Fricks IP, et al. UDP is a competitive antagonist at the human P2Y14 receptor. J Pharmacol Exp Ther. 2008;325(2):588-594.

45. Schmitz N, et al. Randomised trial of filgrastimmobilised peripheral blood progenitor cell transplantation versus autologous bone-marrow transplantation in lymphoma patients. Lancet. 1996;347(8998):353-357.

46. Cottler-Fox MH, et al. Stem cell mobilization. Hematology Am Soc Hematol Educ Program. 2003:419-437.

47. Hornung RL, Longo DL. Hematopoietic stem cell depletion by restorative growth factor regimens during repeated high-dose cyclophosphamide therapy. Blood. 1992;80(1):77-83.

48. Chitteti BR, Liu Y, Srour EF. Genomic and proteomic analysis of the impact of mitotic quiescence on the engraftment of human CD34+ cells. PLoS One. 2011;6(3):e17498.

49. Micklem HS, Anderson N, Ross E. Limited potential of circulating haemopoietic stem cells. Nature. 1975;256(5512):41-43.

50. Schwarzenberger P, et al. Il-17 mobilizes peripheral blood stem cells with short- and long-term repopulating ability in mice. JImmunol. 2001;167(4):2081-2086.

51. LiJM, Giver CR, Lu Y, Hossain MS, Akhtari M, Waller EK. Separating graft-versus-leukemia from graft-versus-host disease in allogeneic hematopoietic stem cell transplantation. Immunotherapy. 2009;1(4):599-621.

52. Lekli I, Gurusamy N, Ray D, Tosaki A, Das DK. Redox regulation of stem cell mobilization. Can J Physiol Pharmacol. 2009;87(12):989-995.

53. Pruijt JF, et al. Neutrophils are indispensable for hematopoietic stem cell mobilization induced by interleukin-8 in mice. Proc Natl Acad Sci U S A. 2002;99(9):6228-6233.

54. Brautigam VM, Dubyak GR, Crain JM, Watters JJ. The inflammatory effects of UDP-glucose in N9 microglia are not mediated by $\mathrm{P} 2 \mathrm{Y} 14$ receptor activation. Purinergic Signal. 2008;4(1):73-78.

55. Scrivens M, Dickenson JM. Pharmacological effects mediated by UDP-glucose that are independent of P2Y14 receptor expression. Pharmacol Res. 2005;51(6):533-538

56. Liu F, Poursine-Laurent J, Link DC. Expression of the G-CSF receptor on hematopoietic progenitor cells is not required for their mobilization by G-CSF. Blood. 2000;95(10):3025-3031.

57. Greenbaum AM, Link DC. Mechanisms of G-CSFmediated hematopoietic stem and progenitor mobilization. Lenkemia. 2011;25(2):211-217.

58. Lee BC, et al. P2Y-like receptor, GPR105 (P2Y14), identifies and mediates chemotaxis of bone-marrow hematopoietic stem cells. Genes Dev. 2003;17(13):1592-1604.

59. Passegue E, Wagers AJ, Giuriato S, Anderson WC, Weissman IL. Global analysis of proliferation and cell cycle gene expression in the regulation of hematopoietic stem and progenitor cell fates. J Exp Med. 2005;202(11):1599-1611.

60. Ko H, Fricks I, Ivanov AA, Harden TK, Jacobson KA. Structure-activity relationship of uridine 5 '-diphosphoglucose analogues as agonists of the human P2Y14 receptor. J Med Chem. 2007;50(9):2030-2039.

61. Ploemacher RE, van der Sluijs JP, Voerman JS, Brons NH. An in vitro limiting-dilution assay of long-term repopulating hematopoietic stem cells in the mouse. Blood. 1989;74(8):2755-2763.

62. Cho J, et al. Ewing sarcoma gene Ews regulates hematopoietic stem cell senescence. Blood. 2011; 117(4):1156-1166. 